\title{
Compliance of RCTs in endodontic instrumentation with the CONSORT Statement:
}

\section{A systematic review}

\author{
Conformidade de ECRs em instrumentação endodôntica com a declaração CONSORT: Uma \\ revisão sistemática \\ Cumplimiento de los ECAs en instrumentación endodóntica con la Declaración CONSORT: Una \\ revisión sistemática
}

Received: 06/18/2021 | Reviewed: 06/29/2021 | Accept: 07/03/2021 | Published: 07/15/2021

\author{
Karla Aguiar Cabral \\ ORCID: https://orcid.org/0000-0003-0438-9001 \\ Faculdade Paulo Picanço, Brazil \\ E-mail: karlacabral20@gmail.com \\ Ana Cristina Rodrigues Martins \\ ORCID: https://orcid.org/0000-0002-9082-0402 \\ Faculdade Paulo Picanço, Brazil \\ E-mail: aniniha_acrm@hotmail.com \\ Letícia Maíra Wambier \\ ORCID: https://orcid.org/0000-0002-9696-0406 \\ Universidade Positivo, Brazil \\ E-mail: lemwambier@hotmail.com \\ Nilton Vivacqua-Gomes \\ ORCID: https://orcid.org/0000-0002-0096-9802 \\ São Leopoldo Mandic, Brazil \\ E-mail: profesornilton@yahoo.com.br \\ Márcia Rezende \\ ORCID: https://orcid.org/0000-0001-8474-5656 \\ Faculdade Paulo Picanço, Brazil \\ E-mail: marcia.rezende@ facpp.edu.br \\ Alessandra Reis \\ ORCID: https://orcid.org/0000-0001-6798-2094 \\ Universidade Estadual de Ponta Grossa, Brazil \\ E-mail: reis_ale@hotmail.com \\ Juliana Larocca de Geus \\ ORCID: https://orcid.org/0000-0001-9633-0474 \\ Faculdade Paulo Picanço, Brazil \\ E-mail: juliana.degeus@facpp.edu.br
}

\begin{abstract}
This systematic review aimed to evaluate the adherence to the CONSORT Statement in clinical studies comparing different instrumentation techniques in Endodontics. Methodology: A systematic search was carried out through Medline databases. There was no restriction on publication year or idiom. Solely randomized controlled trials (RCTs) were included. The articles were evaluated in compliance with CONSORT. Descriptive analyses of the number of studies by journal, country, and quality assessments were performed with RoB 2.0 Cochrane Collaboration's tool for assessing risk of bias in RCTs. A total of 6779 studies were identified, and only 33 meet the eligibility criteria. In relation to the overall risk of bias, eight out of 33 were classified as at 'low' risk of bias. The overall CONSORT score for the included studies in this review was $22 \pm 6.2$ points. Significant differences among countries were observed (p $=0.03)$. The range of years had a significant influence on the average CONSORT score $(p=0.01)$. The adherence of RCTs of endodontic instrumentation to the CONSORT Statement requires improvements. Adherence to the CONSORT statement will also reduce the high or some concerns risk of bias of studies in the field. CRD42020160552 Keywords: Endodontics; Dental instruments; Randomized controlled trials as topic.
\end{abstract}

\section{Resumo}

Esta revisão sistemática teve como objetivo avaliar a adesão à Declaração CONSORT em estudos clínicos comparando diferentes técnicas de instrumentação em Endodontia. Metodologia: Foi realizada uma busca sistemática nas bases de dados Medline. Não houve restrição quanto ao ano ou idioma de publicação. Apenas ensaios clínicos randomizados (ECRs) foram incluídos. Os artigos foram avaliados de acordo com o CONSORT. Análises descritivas do número de estudos por periódico, país e avaliações de qualidade foram realizadas com a ferramenta RoB 2.0 
Cochrane Collaboration para avaliar o risco de viés em ECRs. Um total de 6.779 estudos foram identificados, e apenas 33 atendem aos critérios de elegibilidade. Em relação ao risco geral de viés, oito de 33 foram classificados como de risco "baixo" de viés. A pontuação geral CONSORT para os estudos incluídos nesta revisão foi de $22 \pm 6,2$ pontos. Foram observadas diferenças significativas entre os países $(p=0,03)$. O intervalo de anos teve influência significativa no escore CONSORT médio $(\mathrm{p}=0,01)$. A adesão dos ECRs de instrumentação endodôntica à declaração CONSORT requer melhorias. A adesão à declaração CONSORT também reduzirá o risco elevado ou algumas preocupações de viés dos estudos na área. CRD42020160552

Palavras-chave: Endodontia; Instrumentos odontológicos; Ensaios clínicos controlados aleatórios como assunto.

\section{Resumen}

Esta revisión sistemática tuvo como objetivo evaluar la adherencia a la Declaración CONSORT en estudios clínicos comparando diferentes técnicas de instrumentación en Endodoncia. Metodología: Se realizó una búsqueda sistemática a través de las bases de datos de Medline. No hubo restricciones sobre el año de publicación o el idioma. Se incluyeron únicamente ensayos controlados aleatorios (ECAs). Los artículos fueron evaluados de acuerdo con CONSORT. Se realizaron análisis descriptivos del número de estudios por revista, país y evaluaciones de calidad con la herramienta de la Colaboración Cochrane RoB 2.0 para evaluar el riesgo de sesgo en los ECAs. Se identificaron un total de 6779 estudios y solo 33 cumplen los criterios de elegibilidad. En relación con el riesgo general de sesgo, ocho de los 33 se clasificaron como de riesgo "bajo" de sesgo. La puntuación CONSORT general para los estudios incluidos en esta revisión fue de $22 \pm 6,2$ puntos. Se observaron diferencias significativas entre países $(\mathrm{p}=0,03)$. El rango de años tuvo una influencia significativa en el puntaje CONSORT promedio $(\mathrm{p}=0.01)$. La adherencia de los ECAs de instrumentación endodóntica a la Declaración CONSORT requiere mejoras. La adherencia a la declaración CONSORT también reducirá el riesgo alto o algunas preocupaciones de sesgo de los estudios en el campo. CRD42020160552

Palabras clave: Endodoncia; Instrumentos dentales; Ensayos clínicos controlados aleatorios como asunto.

\section{Introduction}

Root canal treatment aims to restore a tooth and its underlying tissues to normal condition and function (Mohammadi et al. 2017). It is performed in several interdependent and continuous stages, in which the step of biomechanical preparation of the root canal should clean and remodel the endodontic canal system before obturation procedures (Chugal et al. 2017).

Until the 1960s, endodontic instrumentation was made by serial or conventional technique, using stainless steel instruments in an increasing order of diameter, maintaining the same working length. This protocol presents a higher risk of accidents, especially in curved and overlapped channels (Tortini et al. 2007). Over time, instruments with greater flexibility were developed. They were recommended to be used throughout the working length, while more calibrated instruments, would be used at lower working lengths. This was the rationale being the step-back technique, which significantly reduced accidents when compared to the conventional technique (Kfir et al. 2003).

In 1988, Marshall and Pappin described the Oregon technique, based on the concept of crown-down instrumentation, that is, the preparation is initiated by the cervical third of the canal, followed by the middle third and finally the third apical, where the files would work without producing pressure and minimizing the debris' extrusion to the apical foramen (Marshall \& Pappin 1988).

In order to decrease the working time required for root canal shaping, the employment of nickel-titanium rotary instruments was proposed. These instruments were intended to replace the Gates Glidden drills and hand-files previously used since they present greater flexibility and, consequently, more safety when in use, due to the lower risk of fracture of the instrument. They are instruments used in the enlargement movement in the cervical-apical direction (Del Fabbro et al. 2018). Reciprocating instrumentation emerged after rotary instrumentation, to use a single device produced for single use only. These new systems made the preparation faster, reduced cyclic fatigue of the instruments, and reduced cross-contamination due to their single-use (Jardine et al. 2016).

Various clinical trials have compared different endodontic shaping techniques (Arslan et al. 2016, Comparin et al. 2017, Gathani et al. 2016, Jain et al. 2016, Nekoofar et al. 2015; Neves et al. 2016, Pasqualini et al. 2016), and they led to most of the conclusions clinicians rely on to select the instrumentation system for endodontic treatment. Although randomized 
controlled trials (RCTs) are considered the study design capable of minimizing most of the bias that threatens the quality of the evidence, they only produce reliable evidence if well delineated with good reporting. Incomplete or inadequate reporting of information on the planning and conduction of the study impairs the identification of possible methodological flaws and makes it difficult for the interested parties to use their conclusions since they cannot critically evaluate its clinical applicability (Altman et al. 2001, Schriger \& Altman 2006).

Frequent mistakes in the report of RCTs encouraged a group of editors and researchers to formulate the Consolidated Standards of Reporting Trials Statement (CONSORT) (Altman et al. 2001). This statement assists authors in the reporting process of RCTs in a way to allow readers for a critical interpretation of the study results. Additionally, it prevents the omission of possible systematic errors that would compromise the validity and reliability of the results and, consequently, their applicability within the context of evidence-based dentistry.

Given the importance of RCTs in endodontic instrumentation to make decisions regarding protocols, and commercial brand of instruments, the aim of this study was to systematically review the literature in peer-reviewed journals to evaluate a) the compliance of RCTs with the CONSORT Statement and b) the risk of bias in these RCT studies through the Cochrane Collaboration risk of bias tool.

\section{Methodology}

This is a systematic review of methodology of randomized clinical trials (RCTs) studies (Pereira et al. 2018).

Methodology described here follow the PRISMA Statement and was based on previous systematic reviews of methodology (Loguercio et al. 2017, Reis et al. 2018).

\section{Protocol and registration}

The study was registered at the protocol at the PROSPERO database (CRD42020160552).

\section{Eligibility criteria}

This systematic review included only RCTs published in peer-review journals that compared different endodontic instrumentation techniques in adults.

\section{Information sources}

A search was made in the scientific literature in July 2018, through the Pubmed database (primary search, Table 1), which was transferred and adapted to the other databases (Scopus, Web of Science, LILACS, BBO and Cochrane), without restriction of date or language of publication. 
Table 1. Search strategy for Pubmed.

\begin{tabular}{|c|c|c|}
\hline \multicolumn{3}{|l|}{ PUBMED 06/08/2018 } \\
\hline$\# 1$ & \#2 & $\# 3$ \\
\hline 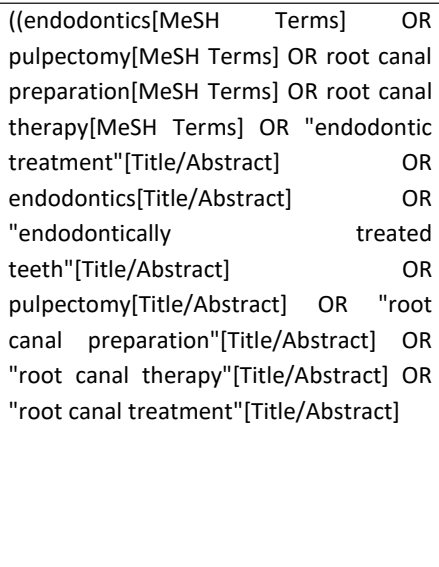 & 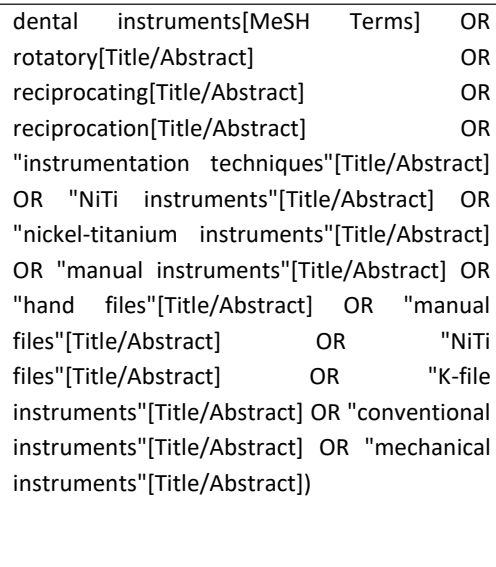 & $\begin{array}{l}\text { (randomized controlled trial[pt] OR } \\
\text { controlled clinical trial[pt] OR randomized } \\
\text { controlled trials[mh] OR random } \\
\text { allocation[mh] OR double-blind method[mh] } \\
\text { OR single-blind method[mh] OR clinical } \\
\text { trial[pt] OR clinical trials[mh] OR ("clinical } \\
\text { trial"[tw]) OR ((singl*[tw] OR doubl*[tw] OR } \\
\text { trebl*[tw] OR tripl*[tw]) AND (mask*[tw] OR } \\
\text { blind*[tw])) OR (placebos[mh] OR } \\
\text { placebo*[tw] OR random*[tw] OR research } \\
\text { design[mh:noexp] OR comparative study[pt] } \\
\text { OR evaluation studies as topic[mh] OR } \\
\text { follow-up studies[mh] OR prospective } \\
\text { studies[mh] OR control*[tw] OR } \\
\text { prospective*[tw] OR volunteer*[tw]) NOT } \\
\text { (animals[mh] NOT humans[mh]))) }\end{array}$ \\
\hline \#1 AND \#2 AND \#3 & & \\
\hline
\end{tabular}

Source: Authors.

\section{Study selection}

The search strategy was based on the concepts of participant and intervention in which the participant comprised patients in need of endodontic treatment, and the intervention involved terms that included rotary instrumentation, at least in one of the groups of comparisons. Initially, the search strategy was elaborated for PubMed. For this purpose, Mesh terms and keywords were used, combined with the Boolean operator OR within each concept, and the concepts were combined with the Boolean operator AND. Then, this search strategy was adapted to the other databases described earlier.

After running the search strategy, a reference management program was used (EndNote X6, Clarivate Analytics, Philadelphia, PA, USA) to store the files of all databases. Then, the duplicate articles were removed using a software tool, and this was also followed by manual removal after the organization of titles in alphabetical order. The relevance of the articles was screened by titles, abstracts, and finally, with the complete full-text of the remaining articles. The full-texts were obtained and read to check if they met the inclusion and exclusion criteria. All these steps were performed independently by two authors (J.L.G. and L.M.W.), who reached a consensus whenever disagreements occurred.

\section{Data collection process}

Two authors collected data related to the study design, study setting, number of participants per group, age of participants, percentage of male, endodontic instruments used, instrumentation protocol, anesthetic technique, anesthetic salt and amount used, tooth endodontic treatment, number of sessions, intracanal medication used, irrigation solution, obturation technique and endodontic cement, sample losses and evaluated outcomes were collected by two authors (J.L.G. and L.M.W.). If the study evaluated postoperative pain, the criteria for pain evaluation, and the assessment times were collected.

\section{Risk of bias in individual studies}

The Cochrane Collaboration's recommended tool for assessing risk of bias (RoB) is neither a scale nor a checklist. It is a domain-based evaluation, in which critical assessments are made separately for different domains. The RoB of the 
individual studies was evaluated by two independent evaluators (J.L.G. and L.M.W.) using the RoB version 2.0, a revised tool to assess the risk of bias in RCTs. This tool evaluated the following items: bias arising from the randomization process, bias due to deviations from intended interventions, bias due to missing outcome data, bias in measurement of the outcome, bias in selection of the reported result, and overall bias.

Risk of bias judgments followed from answers to signaling questions: 'low risk of bias', 'some concerns' or 'high risk of bias'. Overall risk of bias judgment: the study was considered to be at 'low risk of bias' if it was at 'low risk' for all domains, the study was considered to be at 'some concerns' if it was judged to be at 'some concerns' in at least one domain and the study was considered to be at 'high risk of bias' if it was at 'high risk of bias' in at least one domain.

During data selection and quality assessment, any disagreements between the reviewers were solved through discussion, and if needed, by consulting a third reviewer (A.R.).

\section{Adherence to CONSORT statement}

An evaluation tool based on the items related to the methods and results from the 2010 CONSORT Statement (Schulz et al. 2010) was used to evaluate the reporting completeness of RCTs (Table 2). This evaluation tool was adapted from previous publications to match the research question of this investigation (Loguercio et al. 2017, Reis et al. 2018). The items related to the title and abstract, introduction, and discussion were not included since their evaluation is very subjective, and the adherence to these items would not weaken the quality of the study report or the risk of bias of the studies.

A total of 12 items of the CONSORT Statement were included in this CONSORT evaluation tool. As some of these items were subdivided, a total of 16 items was evaluated. The given score per item ranged from 0 to 2 . In general words, $0=$ no description, 1 = poor description and 2 = adequate description. More details regarding the scoring process for each score of each item are displayed in Table 2. Each item was given an equal weight (Loguercio et al. 2017, Reis et al. 2018).

Two reviewers (J.L.G. and L.M.W.) performed the round of scoring using the CONSORT evaluation tool as a guide (Table 2). In case of disagreement, a discussion followed, and the consensus was used to determine the final score. Evaluators were not blinded to the authors of the studies. Unfortunately, this blinding would not be feasible, as reviewers were familiar with the studies and could easily guess the researchers' affiliation by reading the paper.

Table 2. Instrument tool developed from the 2010 CONSORT Statement to evaluate the compliance of the studies to the CONSORT Statement.

\begin{tabular}{|c|c|c|c|}
\hline \multirow{2}{*}{$\begin{array}{l}\text { CONSORT } \\
\text { item }\end{array}$} & \multirow{2}{*}{ Sub-item } & \multirow{2}{*}{ Score } & Adherence to the methods and result items of the consort statement \\
\hline & & & Description \\
\hline \multirow[b]{3}{*}{ Trial design } & & Positive [2] & The trial design is clearly written in the text (split mouth, cross-over, factorial, cluster). \\
\hline & & Negative [0] & This information is not reported. \\
\hline & & Poor [1] & $\begin{array}{l}\text { 1. Information can be obtained by reading the manuscript, although this is not explicity reported by the } \\
\text { authors. } 2 \text {. There is lack of consistence between sections of the article (examples - abstract does not match } \\
\text { the material and methods section; the presentation of the results does not match the description of the trial } \\
\text { design; flow diagram presents different information, etc.). }\end{array}$ \\
\hline \multirow{3}{*}{ Participants } & \multirow{3}{*}{ Eligibility criteria } & Positive [2] & $\begin{array}{l}\text { The inclusion and exclusion criteria is clear, so that readers can know exactly which population the data } \\
\text { can be extrapolated to. }\end{array}$ \\
\hline & & Negative [0] & The information is not reported. \\
\hline & & Poor [1] & $\begin{array}{l}\text { 1. Incomplete information of eligibility criteria compared to most of the studies in the field. } 2 \text {. Presence of } \\
\text { inconsistencies in the inclusion/exclusion criteria that prevents the readers from knowing the population } \\
\text { in which the intervention/control groups were performed. }\end{array}$ \\
\hline
\end{tabular}


Research, Society and Development, v. 10, n. 8, e41910817350, 2021

(CC BY 4.0) | ISSN 2525-3409 | DOI: http://dx.doi.org/10.33448/rsd-v10i8.17350

\begin{tabular}{|c|c|c|c|}
\hline \multirow{5}{*}{$\begin{array}{l}\text { CONSORT } \\
\text { item }\end{array}$} & \multirow{2}{*}{ Sub-item } & \multirow{2}{*}{ Score } & \multirow[t]{2}{*}{ Adherence to the methods and result items of the consort statement } \\
\hline & & & \\
\hline & \multirow{3}{*}{$\begin{array}{l}\text { Settings } \\
\text { location }\end{array}$} & Positive [2] & $\begin{array}{l}\text { Clear description of the setting (academic, practice-based research, university, private clinics, etc.) as well } \\
\text { as the date on which the intervention was implemented. }\end{array}$ \\
\hline & & Negative [0] & The setting and/or the location is not reported in the text. \\
\hline & & Poor [1] & $\begin{array}{l}\text { 1. Authors describe either the setting or the date but never both. 2. This information can be obtained } \\
\text { indirectly in the text }\end{array}$ \\
\hline \multirow{3}{*}{ Interventions } & & Positive [2] & $\begin{array}{l}\text { The interventions for each group are described with sufficient details to allow replication, including how } \\
\text { they were actually administered. }\end{array}$ \\
\hline & & 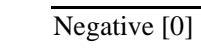 & There is no description. \\
\hline & & Poor [1] & There is missing information that prevents the replication of the interventions/comparators. \\
\hline \multirow{3}{*}{ Outcomes } & & Positive [2] & $\begin{array}{l}\text { At least the primary outcomes were defined in details, including how and when they were assessed. } \\
\text { Consider it as clear when the details are clear, but the authors did not use the term "primary outcome" or } \\
\text { related synonyms. }\end{array}$ \\
\hline & & 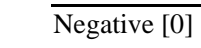 & There is no definition of the primary outcome and/or secondary outcomes. \\
\hline & & Poor [1] & $\begin{array}{l}\text { 1. The authors only report they have used specific criteria without detailing the most important outcomes } \\
\text { of such criteria. } 2 \text {. The description of the primary outcome and/or secondary outcomes is very superficial } \\
\text { and does not allow replication of the method. }\end{array}$ \\
\hline \multirow[t]{3}{*}{ Sample size } & & Positive [2] & $\begin{array}{l}\text { Method of sample size calculation is described in a way to allow replication. It should identify the } \\
\text { primary outcome for each sample size calculated. Elements of the sample size calculation are (1) the } \\
\text { estimated outcomes in each group (which implies the clinically important target difference between the } \\
\text { intervention groups); (2) the } \alpha \text { (type I) error level; (3) the statistical power (or the } \beta \text { (type II) error level); } \\
\text { and (4), for continuous outcomes, the standard deviation of the measurements should be reported. For } \\
\text { equivalence trials, the equivalence limit, instead of the effect size should be reported. }\end{array}$ \\
\hline & & 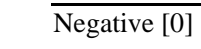 & There is no description in the article. \\
\hline & & Poor [1] & The sample size is described but some parameters are missing so that it prevents replication. \\
\hline \multirow{6}{*}{ Randomization } & \multirow{3}{*}{$\begin{array}{l}\text { Sequence } \\
\text { generation }\end{array}$} & Positive [2] & $\begin{array}{l}\text { 1. Clear description of the random sequence generation. 2. or clear description of a non-random sequence } \\
\text { method. }\end{array}$ \\
\hline & & Negative [0] & There is no information in the text. \\
\hline & & Poor [1] & $\begin{array}{l}\text { The authors only provide a very superficial description (such as the "groups were randomly allocated") or } \\
\text { do not provide sufficient information to allow replication of the randomization process. }\end{array}$ \\
\hline & \multirow{3}{*}{$\begin{array}{l}\text { Allocation } \\
\text { concealment }\end{array}$} & Positive [2] & Clear description of the allocation concealment. See next columns for evaluation of the Risk of Bias. \\
\hline & & Negative [0] & There is no information in the text. \\
\hline & & Poor [1] & not applicable \\
\hline \multirow[t]{3}{*}{ Blinding } & & Positive [2] & $\begin{array}{l}\text { 1) The authors describe who is blinded in the study. 2. In single-blind studies (when this is clearly } \\
\text { reported by the authors), just the description of participant or evaluator (the one blinded) is enough; } \\
\text { however, when the study is double blind or triple blind all blinded people should be described. 2) The } \\
\text { study describes just the participant or examiner blinded but one of these people cannot be blinded due to } \\
\text { the intrinsic features of the study design. }\end{array}$ \\
\hline & & $\begin{array}{l}\text { Negative [0] } \\
\text { na }\end{array}$ & There is no description of the blinding. \\
\hline & & Poor [1] & $\begin{array}{l}\text { Insufficient/partial information. For instance, (1) the authors describe examiners' blinding or participants' } \\
\text { blinding, but never both. (2) The authors describe the study was blind or double-blind but do not specify } \\
\text { who was blinded. }\end{array}$ \\
\hline \multirow[t]{2}{*}{$\begin{array}{l}\text { Statistical } \\
\text { methods }\end{array}$} & \multirow[t]{2}{*}{$\begin{array}{l}\text { Hypothesis } \\
\text { testing }\end{array}$} & Positive [2] & $\begin{array}{l}\text { Statistical methods are described with enough detail to enable a knowledgeable reader with access to the } \\
\text { original data to verify the reported results. Additionally, statistical tests employed by the authors seem to } \\
\text { be adequate for the type of trial design and nature of the data collected. }\end{array}$ \\
\hline & & $\overline{\text { Negative [0] }}$ & tistical methods are not described. \\
\hline
\end{tabular}


Research, Society and Development, v. 10, n. 8, e41910817350, 2021

(CC BY 4.0) | ISSN 2525-3409 | DOI: http://dx.doi.org/10.33448/rsd-v10i8.17350

\begin{tabular}{|c|c|c|c|}
\hline \multirow{6}{*}{$\begin{array}{l}\text { CONSORT } \\
\text { item }\end{array}$} & \multirow{3}{*}{ Sub-item } & \multirow{2}{*}{ Score } & Adherence to the methods and result items of the consort statement \\
\hline & & & cription \\
\hline & & Poor [1] & $\begin{array}{l}\text { 1) There is not enough information to evaluate the statistical method used by the authors and/or the type } \\
\text { of statistical tests employed by the authors are inadequate for the trial design and/or nature of the data (for } \\
\text { instance, tests that do not take into account the paired nature of the data when this is the case). 2) The } \\
\text { authors describe several statistical tests but do not specify each outcome they were applied to. }\end{array}$ \\
\hline & Estimated effect & Positive [2] & $\begin{array}{l}\text { Authors report at least for the primary outcome the effect size and its precision (such as } 95 \% \text { confidence } \\
\text { interval). Odds ratio, risk ratio, risk difference, mean difference, etc. }\end{array}$ \\
\hline & size & 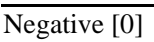 & There is no description of the effect size and $95 \%$ confidence interval \\
\hline & & 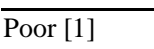 & There is incomplete information. \\
\hline \multirow{6}{*}{$\begin{array}{l}\text { Participant } \\
\text { flow }\end{array}$} & \multirow{3}{*}{ Flow diagram } & Positive [2] & $\begin{array}{l}\text { For each group, the number of participants who were randomly assigned, received intended treatment and } \\
\text { were analyzed for the primary outcome is described in the flow chart CONSORT diagram. }\end{array}$ \\
\hline & & $\begin{array}{l}\text { Negative [0] } \\
\text { ne }\end{array}$ & The flow-chart is not presented in the article. \\
\hline & & Poor [1] & $\begin{array}{l}\text { 1. There are inconsistencies between the numbers described in the flow-chart and other parts of the } \\
\text { manuscript. 2. Incomplete diagram with missing information }\end{array}$ \\
\hline & \multirow{3}{*}{ Losses/Exclusions } & Positive [2] & $\begin{array}{l}\text { 1. For each group, losses and exclusions after randomization are described with reasons. 2. During } \\
\text { reading, reviewer observes that there is no loss to follow-up. }\end{array}$ \\
\hline & & Negati & There is no description of losses and exclusions. \\
\hline & & Poor [1] & $\begin{array}{l}\text { Incomplete information. For instance, } 1 \text {. the authors describe the overall percentage of losses but this } \\
\text { information is not specified per group. } 2 \text {. The authors describe the losses and exclusions but do not } \\
\text { specify the reasons }\end{array}$ \\
\hline \multirow{3}{*}{ Baseline data } & & Positive [2] & $\begin{array}{l}\text { A table/text description containing baseline demographic and clinical characteristics of each group is } \\
\text { presented in the article. }\end{array}$ \\
\hline & & Negative [0] & There is no table/text description with baseline data or description in the body of the text. \\
\hline & & Poor [1] & $\begin{array}{l}\text { 1. A table/ text description with baseline data is presented but the data is not distributed between the study } \\
\text { groups and/or given in percentages instead of raw numbers. 2. Insufficient information about participants } \\
\text { is provided; 3. Inconsistencies in the data presented can be observed. }\end{array}$ \\
\hline \multirow{3}{*}{$\begin{array}{l}\text { Numbers } \\
\text { analysed }\end{array}$} & & Positive [2] & $\begin{array}{l}\text { For each group and for each outcome, the number or participants (denominator) included in the analysis } \\
\text { is clear. }\end{array}$ \\
\hline & & Negative [0] & Authors do not report the numbers analyzed. \\
\hline & & Poor [1] & $\begin{array}{l}\text { There is no clear description of the number of participants (denominator) included in the analysis of at } \\
\text { least one of the outcomes. } 2 \text {. Instead of reporting the raw number of participants, the authors report their } \\
\text { data in percentages. } 3 \text {. The authors fail to report the baseline number of patients included in each analysis. } \\
\text { 4. Data can be obtained indirectly in the study. }\end{array}$ \\
\hline \multirow{3}{*}{$\begin{array}{l}\text { Registration } \\
\text { and protocol }\end{array}$} & & Positive [2] & The study was registered in a trial registry and the protocol number is provided. \\
\hline & & Negative [0] & $\begin{array}{l}\text { This information is not available in the manuscript. Registration in an Ethics Committee is valid as trial } \\
\text { registry }\end{array}$ \\
\hline & & Poor [1] & $\begin{array}{l}\text { The authors describe that the study was registered but does not provide the registration number and/or the } \\
\text { number provided does not link to the study. }\end{array}$ \\
\hline
\end{tabular}

Source: Authors.

\section{Scoring system and statistical analysis}

The number of studies by journal, follow-up period, and country were analyzed descriptively. Compliance with individual items of the CONSORT Statement was analyzed to identify areas in which authors could improve the description. A chart with the percentage of studies per score in each item was provided.

To achieve an overall compliance score, the scores for the 16 items were summed in each article. A trial with 
adequate descriptions (score 2) for all CONSORT items would receive a maximum score of 32 units. A mean average score was calculated by period, journal, and country. Comparison within each factor was performed with the one-way ANOVA and Tukey's test at 95\%. Linear correlation analysis between 2015 ISI journal impact factor, and the average CONSORT score was also performed.

\section{Results}

\section{Study selection}

A total of 6779 studies were identified in the databases. After removing duplicates, 4898 articles remained. With the reading of the titles, this number lowered to 872 , and after abstract screening the number reduced to 39 . Some articles were still excluded for some reasons: five of them were not RCTs (Bartols et al. 2016, Darda et al. 2009, Ritt et al. 2012, Sanfelice et al. 2010, Wan et al. 2017), and one did not compare different instrumentation techniques (Kfir et al. 2006). In summary, only 33 studies remained for assessment (Figure 1).

Figure 1. Flow diagram of study identification.

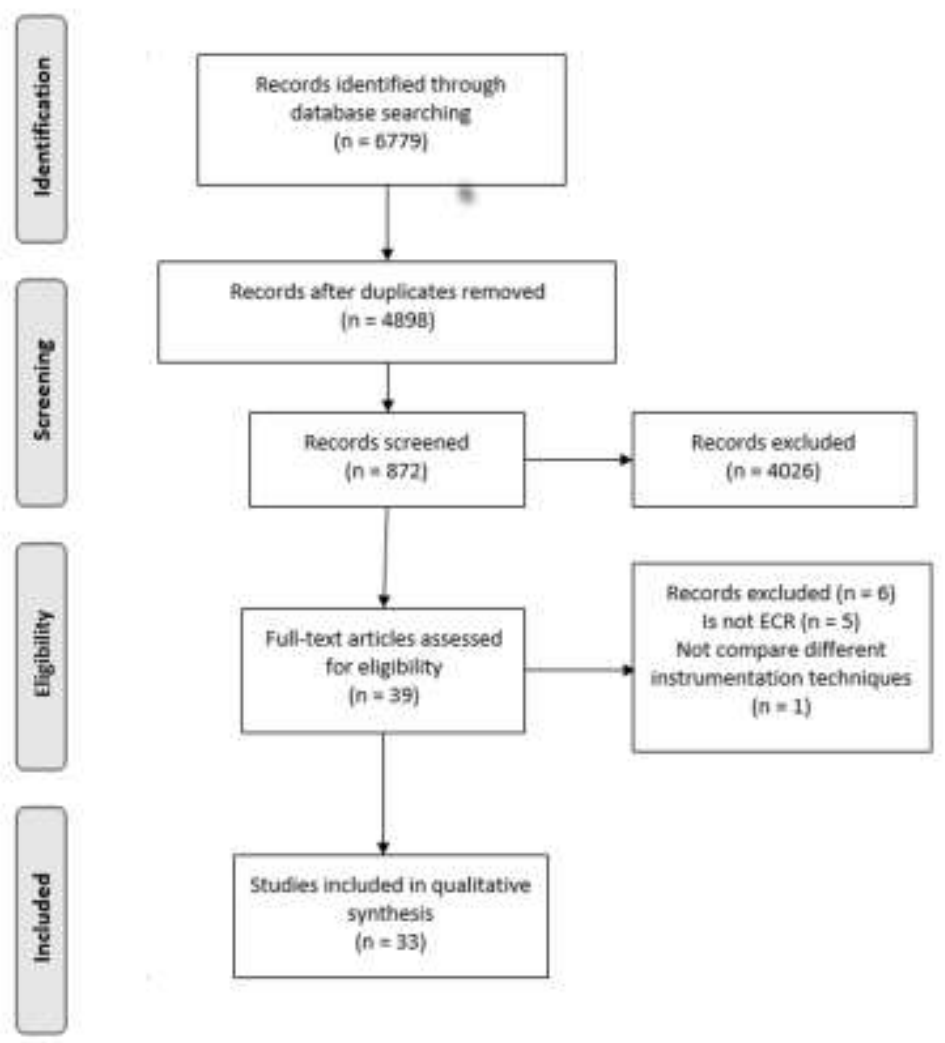

Source: Authors.

\section{Studies characteristics}

The characteristics of the 33 included studies are described in Table 3 and Table 4. 
Table 3. Summary of the studies selected for this systematic review.

\begin{tabular}{|c|c|c|c|c|c|c|c|c|}
\hline Study ID & Journal & $\begin{array}{l}\text { CONSORT } \\
\text { SCORE }\end{array}$ & $\begin{array}{c}\text { Study } \\
\text { design } \\
\text { [Setting] }\end{array}$ & $\begin{array}{c}\text { Subjects' } \\
\text { age in } \\
\text { mean } \pm \\
\text { SD } \\
\text { [range] } \\
\text { (yrs) }\end{array}$ & $\begin{array}{c}\text { \# of Male } \\
\text { [total]/Dro } \\
\text { p-outs }\end{array}$ & $\begin{array}{c}\text { Groups } \\
\text { [\# of } \\
\text { subjects/teeth]/ } \\
\text { Instrumentation } \\
\text { protocol }\end{array}$ & Treated teeth & Pulp condition \\
\hline $\begin{array}{l}\text { Aminsobhani } \\
\text { et al. } 2017\end{array}$ & $\begin{array}{l}\text { Eur } \\
\text { Endod J }\end{array}$ & 28 & $\begin{array}{l}\text { Parallel } \\
\text { [Universit } \\
\text { y] }\end{array}$ & $\begin{array}{c}\text { n.r. } \pm \text { n.r. } \\
{[15-} \\
55]\end{array}$ & $49[105] / 0$ & $\begin{array}{c}\text { NEONITI A1 }{ }^{\mathrm{a}}[21] / \\
\# 25 \\
\left.\text { RACEb }^{2} 21\right] / \\
\# 25 / .06 \\
\text { MTWO }^{\mathrm{c}}[21] / \\
\# 25 / .26 \\
\text { EASY RACE }{ }^{\mathrm{b}}[21] / \\
\# 40 / .10 \text { - \#35/.08 - } \\
\# 25 / .06 \\
\text { MTWO }[21] / \\
\# 10 / .04-\# 15 / .05- \\
\# 20 / .06 \text { - \#25/.06 }\end{array}$ & $\begin{array}{c}\text { Maxillary or } \\
\text { mandibular } \\
\text { molar/premol } \\
\text { ar }\end{array}$ & $\begin{array}{c}\text { Assymptomatic } \\
\text { irreversible } \\
\text { pulpitis }\end{array}$ \\
\hline $\begin{array}{c}\text { Aqrabawi \& } \\
\text { Jamani } \\
2006\end{array}$ & $\begin{array}{c}\text { Odonto } \\
\text { Stomat } \\
\text { ol } \\
\text { Tropica } \\
\text { le }\end{array}$ & 11 & $\begin{array}{c}\text { Tooth as } \\
\text { unit } \\
\text { [Universit } \\
\text { y] }\end{array}$ & $\begin{array}{c}40 \pm \text { n.r. } \\
{[18-} \\
60]\end{array}$ & $64[146] / 0$ & $\begin{array}{c}\text { MANUAL K- } \\
\text { FLEXOFILE }^{\mathrm{d}} \\
\text { [80]/ step-back } \\
\text { technique } \\
\text { PROTAPER }^{\mathrm{d}}[80] / \\
\text { SX - S1 - S2 - F1 } \\
\text { - F2 - F3 }\end{array}$ & $\begin{array}{c}\text { Maxillary or } \\
\text { mandibular } \\
\text { molar/premol } \\
\text { ar }\end{array}$ & $\begin{array}{c}\text { Irreversible } \\
\text { pulpitis }\end{array}$ \\
\hline $\begin{array}{c}\text { Arias et al. } \\
2015\end{array}$ & J Dent & 17 & $\begin{array}{l}\text { Tooth as } \\
\text { unit [n.r.] }\end{array}$ & $\begin{array}{c}\text { n.r. } \pm \text { n.r. } \\
{[<30-} \\
>50]\end{array}$ & n.r. $[88] / 0$ & $\begin{array}{c}\text { MANUAL K- } \\
\text { FLEXOFILE } \\
\text { [44]/ Gates \#1,2 } 2^{\text {nd }} \\
3 \text { - \#10 up to \#40 } \\
\text { PROTAPER } \\
\text { UNIVERSAL + } \\
\text { GTX }[44] / \# 10 \text { - } \\
\# 15 \text { - } 1 \text { - S2 - } \\
\# 20 / .06 \text { - \#30/.06 } \\
\text { (small canals) / } \\
\# 10 \text { - \#15 - 1 - S2 } \\
\text { - \#30/.08 - \#40/.06 } \\
\text { - \#40/.08 (large } \\
\text { canals) }\end{array}$ & Various & $\begin{array}{l}\text { Vital or } \\
\text { nonvital }\end{array}$ \\
\hline $\begin{array}{l}\text { Arslan et al. } \\
2016\end{array}$ & \begin{tabular}{|} 
J Appl \\
Oral \\
Sci
\end{tabular} & 24 & $\begin{array}{l}\text { Parallel } \\
\text { [Universit } \\
\text { y] }\end{array}$ & $\begin{array}{c}31.1 . \pm \\
11.7 \\
\text { [n.r. }- \\
\text { n.r.] }\end{array}$ & $30[56] / 0$ & $\begin{array}{c}\text { RECIPROC }^{\mathrm{e}} 150^{\circ} \\
\mathrm{CCW}-30^{\circ} \mathrm{CW} \\
{[14]} \\
\text { RECIPROC }^{\mathrm{e}} 270^{\circ} \\
\mathrm{CCW}-30^{\circ} \mathrm{CW}\end{array}$ & $\begin{array}{c}\text { Maxillary or } \\
\text { mandibular } \\
\text { molar }\end{array}$ & $\begin{array}{c}\text { Symptomatic } \\
\text { irreversible } \\
\text { pulpitis }\end{array}$ \\
\hline
\end{tabular}


Research, Society and Development, v. 10, n. 8, e41910817350, 2021

(CC BY 4.0) | ISSN 2525-3409 | DOI: http://dx.doi.org/10.33448/rsd-v10i8.17350

\begin{tabular}{|c|c|c|c|c|c|c|c|c|}
\hline & & & & & & $\begin{array}{c}{[14]} \\
\text { RECIPROC }^{\mathrm{e}} 360^{\circ} \\
\mathrm{CCW}-30^{\circ} \mathrm{CW} \\
{[14]} \\
\text { RECIPROC } \\
\text { CONTINUOUS } \\
\text { ROTATION [14] / } \\
\text { R } 40-\text { palatine } \\
\text { and distal canals } \\
\text { R 25 - other canals }\end{array}$ & & \\
\hline Çiçec 2017 & $\begin{array}{r}\text { J Appl } \\
\text { Oral } \\
\text { Sci }\end{array}$ & 25 & $\begin{array}{c}\text { Parallel } \\
\text { [n.r.] }\end{array}$ & $\begin{array}{c}\beta 7.1 \pm \text { n.r. } \\
{[21-} \\
65]\end{array}$ & n.r. $[90] / 0$ & $\begin{array}{c}\text { HAND-FILE }{ }^{\mathrm{d}}[30] / \\
\text { modified step- } \\
\text { back } \\
\text { WAVE ONE }{ }^{\mathrm{d}}[30] / \\
\text { large } \\
\text { PROTAPER NEXT } \\
\text { [30]/ X1 - X2 - } \\
\text { X3 - X4 }\end{array}$ & $\begin{array}{c}\text { Single root } \\
\text { canal }\end{array}$ & Nonvital \\
\hline $\begin{array}{c}\text { Comparin et } \\
\text { al. } 2017\end{array}$ & J Endod & 26 & $\begin{array}{l}\text { Parallel } \\
\text { [Universit } \\
\text { y] }\end{array}$ & $\begin{array}{c}\text { n.r. } \pm \text { n.r. } \\
{[18-} \\
\text { n.r. }]\end{array}$ & $20[65] / 0$ & $\begin{array}{c}\text { MTWO } \\
\text { RETREATMENT }^{\mathrm{e}} \\
\text { [33]/\#15/.05 - } \\
\text { \#25/.05/ \#30/.05 /\# } \\
\text { 35/.04 / \#40/.04 } \\
\text { RECIRPOC } \\
\text { RETREATMENT } \\
\text { [32]/ R25 / R40 }\end{array}$ & Various & Retreatment \\
\hline $\begin{array}{c}\text { Dalton et al. } \\
1998\end{array}$ & J Endod & 18 & $\begin{array}{l}\text { Parallel } \\
\text { [Universit } \\
\text { y] }\end{array}$ & $\begin{array}{c}\text { n.r. } \pm \text { n.r. } \\
\text { [n.r. }- \\
\text { n.r.] }\end{array}$ & n.r. $[48] / 0$ & $\begin{array}{c}\text { MANUAL K- } \\
\text { FILES }^{\mathrm{d}}[24] / \text { step- } \\
\text { back technique } \\
\text { PROFILE }^{\mathrm{f}}[24] / \# 2 \\
\text { up to \#4 /45 / \#6 / } \\
\# 7 \text { or \#8 }\end{array}$ & $\begin{array}{c}\text { Mandibular } \\
\text { molar/premol } \\
\text { ar }\end{array}$ & $\begin{array}{c}\text { Apical } \\
\text { periodontitis } \\
\text { or pulpal } \\
\text { pathosis }\end{array}$ \\
\hline $\begin{array}{c}\text { Dourado et } \\
\text { al. } 2005\end{array}$ & $\begin{array}{r}\text { J Appl } \\
\text { Oral } \\
\text { Sci }\end{array}$ & 21 & $\begin{array}{l}\text { Parallel } \\
\text { [Universit } \\
\text { y] }\end{array}$ & $\begin{array}{c}27.7 \pm \text { n.r. } \\
{[16-} \\
52]\end{array}$ & $12[50] / 0$ & $\begin{array}{l}\text { MANUAL K- } \\
\text { FILES }^{\mathrm{d}}[25] / \text { step- } \\
\text { back technique } \\
\text { K3 }^{\mathrm{g}}[25] / \text { crown- } \\
\text { down technique }\end{array}$ & $\begin{array}{c}\text { Single root } \\
\text { canal }\end{array}$ & Nonvital \\
\hline $\begin{array}{c}\text { Endo et al. } \\
2014\end{array}$ & $\begin{array}{c}\text { Dental } \\
\text { Press } \\
\text { Endod }\end{array}$ & 19 & $\begin{array}{l}\text { Parallel } \\
\text { [Universit } \\
\text { y] }\end{array}$ & $\begin{array}{c}\text { n.r. } \pm \text { n.r. } \\
{[19-} \\
65]\end{array}$ & n.r. $[30] / 0$ & $\begin{array}{l}\text { MANUAL K- } \\
\text { FILES }^{\mathrm{d}}[15] / \text { step- } \\
\text { back technique } \\
\text { MTWO }^{\mathrm{c}}[15] / \\
\# 15 / .05 \text { - \#25/.05 - } \\
\# 30 / .05 \text { - \#35/.04 - } \\
\# 40 / .04\end{array}$ & n.r. & Retreatment \\
\hline Fava 1995 & nt Endod & 10 & Tooth as & n.r. \pm n.r. & n.r. $[78] / 0$ & MANUAL K- & Central incisors & Nonvital \\
\hline
\end{tabular}


Research, Society and Development, v. 10, n. 8, e41910817350, 2021

(CC BY 4.0) | ISSN 2525-3409 | DOI: http://dx.doi.org/10.33448/rsd-v10i8.17350

\begin{tabular}{|c|c|c|c|c|c|c|c|c|}
\hline & $\mathrm{J}$ & & unit [n.r.] & $\begin{array}{c}{[14-} \\
63]\end{array}$ & & $\begin{array}{l}\text { FILES }^{\mathrm{g}} \text { cervical to } \\
\text { apical [30]/ } \\
\text { cervical to apical } \\
\text { MANUAL K- } \\
\text { FILES }^{\mathrm{g}} \text { crown } \\
\text { down [30]/ crown } \\
\text { down } \\
\text { MANUAL K- } \\
\text { FILESg balanced- } \\
\text { force [30]/ } \\
\text { balanced-force }\end{array}$ & & \\
\hline $\begin{array}{c}\text { Gambarini et } \\
\text { al. } 2017\end{array}$ & Clin Ter & 9 & $\begin{array}{l}\text { Tooth as } \\
\text { unit [n.r.] }\end{array}$ & $\begin{array}{c}47.9 \pm \text { n.r. } \\
{[18-} \\
76]\end{array}$ & n.r. $[50] /$ n.r. & $\begin{array}{c}\text { TF ADAPTATIVE } \\
\text { crown down [50]/ } \\
\# 15-\text { ML1 - ML2 } \\
\text { TF ADAPTATIVE } \\
\text { MIMERACI [50]/ } \\
\text { MI = manual } \\
\text { insertion, ME = } \\
\text { minimal } \\
\text { enlargement, R = } \\
\text { remove, AC = and } \\
\text { clean flutes, } \mathrm{I}= \\
\text { irrigate }\end{array}$ & $\begin{array}{l}\text { Maxillary or } \\
\text { mandibular } \\
\text { molar/premol } \\
\text { ar }\end{array}$ & Nonvital \\
\hline $\begin{array}{c}\text { Gatthani \& } \\
\text { Raghavend } \\
\text { ra } 2016\end{array}$ & Endo & 21 & $\begin{array}{c}\text { split-mouth } \\
\text { [Universit } \\
\text { y] }\end{array}$ & $\begin{array}{c}24.6 \pm \text { n.r. } \\
{[16-} \\
46]\end{array}$ & $16[30] / 6$ & $\begin{array}{c}\text { PROTAPER } \\
\text { UNIVERSAL }^{\mathrm{d}} \\
\text { [20]/ SX - S1 - S2 } \\
-\mathrm{F} 1-\mathrm{F} 2 \\
\text { HERO SHAPER } \\
\text { i } \\
\text { [20]/ \#20/.06 - } \\
\# 20 / .04-\# 25 / .06 \\
\text { SELF-ADJUSTING } \\
\text { FILE }[20] / 1.5 \\
\mathrm{~mm}\end{array}$ & $\begin{array}{c}\text { Maxillary or } \\
\text { mandibular } \\
\text { molar }\end{array}$ & Nonvital \\
\hline $\begin{array}{c}\text { Gomes et al. } \\
2017\end{array}$ & $\begin{array}{c}\text { Restor } \\
\text { Dent } \\
\text { Endod }\end{array}$ & 29 & $\begin{array}{c}\text { Split-mouth } \\
\text { [Universit } \\
y]\end{array}$ & $\begin{array}{c}46 \pm 18 \\
{[25-} \\
69]\end{array}$ & $26[55] / 0$ & $\begin{array}{c}\text { MTWO }^{c}[55] / \\
\# 10 / .04-\# 15 / .05- \\
\# 20 / .06-\# 25 / .06 \\
\text { RECIPROC }{ }^{\mathrm{e}}[55] / \\
\text { R25 }\end{array}$ & $\begin{array}{c}\text { Maxillary or } \\
\text { mandibular } \\
\text { molar }\end{array}$ & $\begin{array}{l}\text { Vital or } \\
\text { nonvital }\end{array}$ \\
\hline $\begin{array}{c}\text { Jain et al. } \\
2016\end{array}$ & Endo & 23 & $\begin{array}{c}\text { Parallel } \\
\text { [Private } \\
\text { office] }\end{array}$ & $\begin{array}{c}36 \pm 14 \\
{[30-} \\
55]\end{array}$ & $77[141] / 0$ & $\begin{array}{c}\text { WAVe ONE }{ }^{\mathrm{d}}[47] / \\
\# 25 / .08 \text { or \#40/.08 } \\
\text { ONE SHAPE }[47] / \\
\# 25 / .06 \\
\text { SELF-ADJUSTING } \\
\text { FILE }^{\mathrm{j}}[47] / 1.5\end{array}$ & $\begin{array}{c}\text { Maxillary or } \\
\text { mandibular } \\
\text { molar }\end{array}$ & $\begin{array}{c}\text { Symptomatic } \\
\text { irreversible } \\
\text { pulpitis }\end{array}$ \\
\hline
\end{tabular}


Research, Society and Development, v. 10, n. 8, e41910817350, 2021

(CC BY 4.0) | ISSN 2525-3409 | DOI: http://dx.doi.org/10.33448/rsd-v10i8.17350

\begin{tabular}{|c|c|c|c|c|c|c|c|c|}
\hline & & & & & & $\mathrm{mm}$ or $2.0 \mathrm{~mm}$ & & \\
\hline $\begin{array}{l}\text { Kashofinejad } \\
\text { et al. } 2016\end{array}$ & $\begin{array}{l}\text { J Dent } \\
\text { (Tehran } \\
\text { ) }\end{array}$ & 24 & $\begin{array}{l}\text { Tooth as } \\
\text { unit } \\
\text { [Universit } \\
\text { y] }\end{array}$ & $\begin{array}{l}31,7 \pm \\
10.4[17 \\
-52]\end{array}$ & n.r. [53]/9 & $\begin{array}{c}\text { MANUAL K- } \\
\text { FILES }^{\mathrm{k}}[30] / \text { step- } \\
\text { back technique } \\
\text { MTWO }^{\mathrm{c}}[30] / \\
\# 15 / .05 \text { - \#20/.06 - } \\
\# 25 / .06 \text { - \#30/.05 - } \\
\# 35 / .04 \text { - \#40/.04 - } \\
\# 25 / .07\end{array}$ & $\begin{array}{c}\text { Single root } \\
\text { canal }\end{array}$ & $\begin{array}{c}\text { Symptomatic } \\
\text { irreversible } \\
\text { pulpitis }\end{array}$ \\
\hline $\begin{array}{l}\text { Kherlakian } \\
\text { et al. } 2016\end{array}$ & J Endod & 27 & $\begin{array}{c}\text { Parallel } \\
\text { [Private } \\
\text { office] }\end{array}$ & $\begin{array}{c}47 \pm \text { n.r. } \\
{[19-} \\
73]\end{array}$ & $77[210] / 0$ & $\begin{array}{c}\text { PROTAPER NEXT } \\
\text { [70]/ SX }-\mathrm{X} 1- \\
\text { X2 }-\mathrm{X} 3-\mathrm{X} 4 \\
\text { WAVE ONE }^{\mathrm{d}}[70] / \\
\text { primary or large } \\
\text { RECIPROC }^{\mathrm{e}}[70] / \\
\text { R25 or R40 }\end{array}$ & $\begin{array}{l}\text { Maxillary or } \\
\text { mandibular } \\
\text { molar/premol } \\
\text { ar }\end{array}$ & $\begin{array}{c}\text { Irreversible } \\
\text { pulpitis }\end{array}$ \\
\hline $\begin{array}{l}\text { Krithikadatta } \\
\text { et al. } 2016\end{array}$ & $\begin{array}{c}\text { Conserv } \\
\text { Dent }\end{array}$ & 29 & $\begin{array}{l}\text { Parallel } \\
\text { [Universit } \\
\text { y] }\end{array}$ & $\begin{array}{c}\text { n.r. } \pm \text { n.r. } \\
{[18-} \\
55]\end{array}$ & $69[152] / 3$ & $\begin{array}{c}\text { WAVE ONE }{ }^{\mathrm{d}}[50] \\
\text { PROTAPER } \\
\text { UNIVERSAL }^{\mathrm{d}} \\
\text { [51] } \\
\text { MTWO }^{\mathrm{c}}[51] / \\
\text { According to } \\
\text { manufactures' } \\
\text { instructions }\end{array}$ & $\begin{array}{l}\text { Maxillary or } \\
\text { mandibular } \\
\text { molar/premol } \\
\text { ar }\end{array}$ & $\begin{array}{l}\text { Vital or } \\
\text { nonvital }\end{array}$ \\
\hline $\begin{array}{l}\text { Mollashahi } \\
\text { et al. } 2017\end{array}$ & $\begin{array}{l}\text { Iran } \\
\text { Endod J }\end{array}$ & 28 & $\begin{array}{l}\text { Parallel } \\
\text { [Universit } \\
\text { y] }\end{array}$ & $\begin{array}{c}31.7 \pm 5.1 \\
{[20-} \\
50]\end{array}$ & $63[150] / 0$ & $\begin{array}{c}\text { MANUAL K- } \\
\text { FILES }[50] / \text { up to } \\
\text { \#25 or up to \#40 } \\
\text { ONE SHAPE }[50] / \\
\# 25 / .06 \\
\text { RECIPROC }{ }^{\mathrm{e}}[50] / \\
\text { R25 or R40 }\end{array}$ & $\begin{array}{c}\text { Maxillary or } \\
\text { mandibular } \\
\text { molar }\end{array}$ & $\begin{array}{c}\text { Symptomatic } \\
\text { irreversible } \\
\text { pulpitis }\end{array}$ \\
\hline $\begin{array}{c}\text { Neelakantan } \\
\text { \& Sharma } \\
2015\end{array}$ & $\begin{array}{l}\text { Clin Oral } \\
\text { Investig }\end{array}$ & 24 & $\begin{array}{c}\text { Tooth as } \\
\text { unit } \\
\text { [Private } \\
\text { office] }\end{array}$ & $\begin{array}{c}\beta 1 \pm 2[25 \\
-40]\end{array}$ & $311[605] / 40$ & $\begin{array}{c}\text { ONE SHAPE }^{\mathrm{i}}[624] \\
\text { RECIPROC }^{\mathrm{e}}[624] / \\
\text { According to } \\
\text { manufactures' } \\
\text { instructions }\end{array}$ & $\begin{array}{c}\text { Mandibular } \\
\text { molar }\end{array}$ & $\begin{array}{c}\text { Symptomatic } \\
\text { irreversible } \\
\text { pulpitis }\end{array}$ \\
\hline $\begin{array}{c}\text { Nekoofar et } \\
\text { al. } 2015\end{array}$ & $\mathrm{~J}$ Endod & 20 & $\begin{array}{l}\text { Parallel } \\
\text { [Universit } \\
\text { y] }\end{array}$ & $\begin{array}{c}\text { n.r. } \pm \text { n.r. } \\
{[15-} \\
55]\end{array}$ & $22[42] / n . r$. & $\begin{array}{c}\text { WAVE ONE }^{\mathrm{d}}[21] / \\
\mathrm{SX}-\mathrm{S} 1-\mathrm{S} 2- \\
\text { F1/F2/F3 } \\
\text { PROTAPER } \\
\text { UNIVERSAL }^{\mathrm{d}} \\
{[21] / \text { small or }} \\
\text { large }\end{array}$ & $\begin{array}{l}\text { Maxillary or } \\
\text { mandibular } \\
\text { molar/premol } \\
\text { ar }\end{array}$ & $\begin{array}{c}\text { Irreversible } \\
\text { pulpitis }\end{array}$ \\
\hline Neves et al. & J Endod & 19 & Parallel & $39 \pm$ n.r. & $20[60] / 1$ & RECIPROC $^{\mathrm{e}}[30] /$ & Single root & Nonvital \\
\hline
\end{tabular}


Research, Society and Development, v. 10, n. 8, e41910817350, 2021

(CC BY 4.0) | ISSN 2525-3409 | DOI: http://dx.doi.org/10.33448/rsd-v10i8.17350

\begin{tabular}{|c|c|c|c|c|c|c|c|c|}
\hline 2016 & & & $\begin{array}{c}\text { [Universit } \\
\mathrm{y}]\end{array}$ & $\begin{array}{c}{[16-} \\
85]\end{array}$ & & $\begin{array}{c}\text { R40 or R50 } \\
\text { BIORACE }[30] / \\
\# 40 / .04 \text { OR } \\
\# 50 / .04\end{array}$ & canal & \\
\hline $\begin{array}{c}\text { Pasqualini et } \\
\text { al. } 2016\end{array}$ & $\begin{array}{c}\text { nt Endod } \\
\mathrm{J}\end{array}$ & 30 & $\begin{array}{l}\text { Parallel } \\
\text { [Universit } \\
\text { y] }\end{array}$ & $\begin{array}{c}\text { n.r. } \pm \text { n.r. } \\
{[16-} \\
60]\end{array}$ & $26[52] / 5$ & $\begin{array}{c}\text { WAVE ONE }{ }^{\mathrm{d}}[26] / \\
\text { S1 - S2 - F1 - F2 } \\
\text { PROTAPER } \\
\text { UNIVERSAL }^{\mathrm{d}} \\
\text { [26]/ primary }\end{array}$ & Various & $\begin{array}{l}\text { Vital or } \\
\text { nonvital }\end{array}$ \\
\hline $\begin{array}{c}\text { Peters et al. } \\
2004\end{array}$ & $\begin{array}{c}\text { nt Endod } \\
\mathrm{J}\end{array}$ & 19 & $\begin{array}{c}\text { Tooth as } \\
\text { unit } \\
\text { [Universit } \\
\text { y] }\end{array}$ & $\begin{array}{c}41.9 \pm \\
13.4 \\
\text { [n.r. - } \\
\text { n.r.] }\end{array}$ & $81[179] / 24$ & $\begin{array}{l}\text {-IGHTSPEED }{ }^{\text {[ }} \text { 48]/ } \\
\text { step-back } \\
\text { technique } \\
\text { PROFILE }{ }^{\mathrm{d}}[103] / \\
\text { step-down and } \\
\text { crown-down .04 } \\
\text { GT }^{\mathrm{d}}[117] / \text { step- } \\
\text { down and crown- } \\
\text { down .04 and .06 }\end{array}$ & Various & $\begin{array}{l}\text { Vital or } \\
\text { nonvital }\end{array}$ \\
\hline $\begin{array}{r}\text { Pettiette et } \\
\text { al. } 2001\end{array}$ & J Endod & 17 & $\begin{array}{l}\text { Parallel } \\
\text { [Universit } \\
\text { y] }\end{array}$ & $\begin{array}{c}\text { n.r. } \pm \text { n.r. } \\
\text { [n.r. }- \\
\text { n.r.] }\end{array}$ & n.r. $[60] / 20$ & $\begin{array}{c}\text { MANUAL K- } \\
\text { FILES }^{\text {n.r. }}[30] / \\
\text { step-back } \\
\text { technique } .02 \\
\text { NITI MANUAL } \\
\text { FILES } \\
\text { str. [30]/ } \\
\text { technique } .02\end{array}$ & $\begin{array}{c}\text { Maxillary or } \\
\text { mandibular } \\
\text { molar }\end{array}$ & Nonvital \\
\hline $\begin{array}{c}\text { Relvas et al. } \\
2016\end{array}$ & $\begin{array}{l}\text { Clin Oral } \\
\text { Investig }\end{array}$ & 31 & $\begin{array}{l}\text { Parallel } \\
\text { [Universit } \\
\text { y] }\end{array}$ & $\begin{array}{c}25.8 \pm 9.2 \\
{[18-} \\
64]\end{array}$ & $78[78] / 0$ & $\begin{array}{c}\text { RECIPROC }{ }^{\mathrm{e}} \text { [39]/ } \\
\text { R25, R40 or R50 } \\
\text { PROTAPER } \\
\text { UNIVERSAL }^{\mathrm{d}} \\
\text { [39]/ SX - S1 - S2 } \\
\text { - F1/F2/F3/F4 }\end{array}$ & $\begin{array}{c}\text { Mandibular } \\
\text { molar }\end{array}$ & Nonvital \\
\hline $\begin{array}{c}\text { Saha et al. } \\
\qquad 2018\end{array}$ & {$\left[\begin{array}{c}\text { Conserv } \\
\text { Dent }\end{array}\right.$} & 24 & $\begin{array}{l}\text { Parallel } \\
\text { [Universit } \\
\text { y] }\end{array}$ & $\begin{array}{c}\text { n.r. } \pm \text { n.r. } \\
{[18-} \\
55]\end{array}$ & n.r. $[214] / 4$ & $\begin{array}{c}\text { PROTAPER NEXT } \\
\text { [72] } \\
\text { WAVE ONE } \\
\text { GOLD }^{\mathrm{d}} \text { [71] } \\
\text { SELF-ADJUSTING } \\
\text { FILE } \text { [71]/ } \\
\text { According to } \\
\text { manufactures' } \\
\text { instructions }\end{array}$ & $\begin{array}{l}\text { Maxillary or } \\
\text { mandibular } \\
\text { molar/premol } \\
\text { ar }\end{array}$ & $\begin{array}{c}\text { Irreversible } \\
\text { pulpitis }\end{array}$ \\
\hline $\begin{array}{l}\text { Saumya- } \\
\text { Rajesh et } \\
\text { al. } 2017\end{array}$ & $\begin{array}{c}\text { nt Endod } \\
\text { J }\end{array}$ & 31 & $\begin{array}{l}\text { Parallel } \\
\text { [Universit } \\
\text { y] }\end{array}$ & $\begin{array}{c}\text { n.r. } \pm \text { n.r. } \\
{[18-} \\
55]\end{array}$ & $60[130] / 3$ & $\begin{array}{c}\text { MTWO }^{\mathrm{c}}[65] / \\
\# 15 / .05-\# 20 / .06- \\
\# 25 / .06-\# 30 / .05 \\
\text { SELF-ADJUSTING }\end{array}$ & $\begin{array}{c}\text { Maxillary or } \\
\text { mandibular } \\
\text { molar/premol } \\
\text { ar }\end{array}$ & $\begin{array}{l}\text { Vital or } \\
\text { nonvital }\end{array}$ \\
\hline
\end{tabular}


Research, Society and Development, v. 10, n. 8, e41910817350, 2021

(CC BY 4.0) | ISSN 2525-3409 | DOI: http://dx.doi.org/10.33448/rsd-v10i8.17350

\begin{tabular}{|c|c|c|c|c|c|c|c|c|}
\hline & & & & & & $\begin{array}{c}\text { FILE }^{\mathrm{J}}[65] / 1.5 \\
\mathrm{~mm}\end{array}$ & & \\
\hline $\begin{array}{c}\text { Shahi et al. } \\
2016\end{array}$ & $\begin{array}{l}\text { Iran } \\
\text { Endod J }\end{array}$ & 27 & $\begin{array}{l}\text { Parallel } \\
\text { [Universit } \\
\text { y] }\end{array}$ & $\begin{array}{c}\text { n.r. } \pm \text { n.r. } \\
{[20-} \\
50]\end{array}$ & n.r. [82]/4 & $\begin{array}{c}\text { RACE }^{\mathrm{d}}[42] / 40 / 0.1 \\
-35 / 0.08-30 / .06 \\
-25 / .04-30 / 0.04 \\
\text { PROTAPER } \\
\text { UNIVERSAL }{ }^{\mathrm{b}} \\
\text { [40]/ SX - S } 1-\mathrm{S} 2 \\
-\mathrm{F} 1-\mathrm{F} 2\end{array}$ & $\begin{array}{c}\text { Mandibular } \\
\text { molar }\end{array}$ & $\begin{array}{c}\text { Irreversible } \\
\text { pulpitis }\end{array}$ \\
\hline $\begin{array}{c}\text { Shokraneh et } \\
\text { al. } 2016\end{array}$ & $\begin{array}{l}\text { Clin Oral } \\
\text { Investig }\end{array}$ & 29 & $\begin{array}{l}\text { Parallel } \\
\text { [Universit } \\
\text { y] }\end{array}$ & $\begin{array}{c}30.5 \pm 4.6 \\
{[20-} \\
45]\end{array}$ & 47 [93]/n.r. & $\begin{array}{c}\text { HAND-FILE }{ }^{\mathrm{d}}[32] / \\
\text { crown-down } \\
\text { technique } \\
\text { WAVE ONE }{ }^{\mathrm{d}}[32] / \\
\text { primary } \\
\text { PROTAPER } \\
\text { UNIVERSAL } \\
\text { [32]/ SX - S1 - S2 } \\
\text { - F1 - F2 }\end{array}$ & $\begin{array}{c}\text { Mandibular } \\
\text { molar }\end{array}$ & Nonvital \\
\hline $\begin{array}{c}\text { Topçuaglu \& } \\
\text { Topçuaglu } \\
2017\end{array}$ & $\begin{array}{l}\text { Acta } \\
\text { Odonto } \\
\text { 1 Scand }\end{array}$ & 21 & $\begin{array}{l}\text { Parallel } \\
\text { [Universit } \\
\text { y] }\end{array}$ & $\begin{array}{c}39.2 \pm 8 \\
{[20-} \\
52]\end{array}$ & $69[135] / 5$ & $\begin{array}{c}\text { HAND-FILE }{ }^{\mathrm{d}}[45] / \\
\# 30 \text { up to \#15 - } \\
\text { \#20 up to \#50 } \\
\text { PROTAPER } \\
\text { UNIVERSAL and } \\
\text { RETREATMENT } \\
\text { d [45]/ D1 - D2 - } \\
\text { D3 - F2 - F3 - F4 } \\
\text { - F5 } \\
\text { RECIPROC }[45] / \\
\text { R25 - R40 }\end{array}$ & $\begin{array}{r}\text { Maxillary } \\
\text { incisor }\end{array}$ & retreatment \\
\hline $\begin{array}{c}\text { Wang et al. } \\
2010\end{array}$ & $\begin{array}{c}\text { Shanghai } \\
\mathrm{J} \\
\text { Estoma } \\
\text { tol }\end{array}$ & 14 & $\begin{array}{l}\text { Parallel } \\
\text { [Universit } \\
\text { y] }\end{array}$ & $\begin{array}{c}\text { n.r. } \pm \text { n.r. } \\
{[22-} \\
71]\end{array}$ & $53[90] /$ n.r. & $\begin{array}{c}\text { K3 }^{\mathrm{g}}[30] / 25 / 0.10- \\
25 / 0.08-25 / 0.06 \\
\text { MTWO }^{\mathrm{c}}[30] / \\
\# 15 / 0.05- \\
\# 20 / 0.06- \\
\# 25 / 0.06 \\
\text { PROTAPER } \\
\text { UNIVERSAL } \\
{[30] / \mathrm{SX}-\mathrm{S} 1-\mathrm{S} 2} \\
-\mathrm{F} 1\end{array}$ & $\begin{array}{c}\text { Maxillary or } \\
\text { mandibular } \\
\text { molar }\end{array}$ & $\begin{array}{l}\text { Vital or } \\
\text { nonvital }\end{array}$ \\
\hline $\begin{array}{c}\text { Wei et al. } \\
2003\end{array}$ & $\begin{array}{c}\text { Hua Xi } \\
\text { Kou } \\
\text { Qiang } \\
\text { Yi Xue } \\
\text { Za Zhi }\end{array}$ & 15 & $\begin{array}{l}\text { Parallel } \\
\text { [Universit } \\
\text { y] }\end{array}$ & $\begin{array}{c}41 \pm \text { n.r. } \\
{[21-} \\
73]\end{array}$ & $32[84] / 0$ & $\begin{array}{l}\text { MANUAL K- } \\
\text { FLEXO FILE }^{\mathrm{d}} \\
\text { [48]/ step-back } \\
\text { technique } \\
\text { PROFILE }{ }^{\mathrm{d}}[47] / \\
\text { according to }\end{array}$ & $\begin{array}{c}\text { Maxillary or } \\
\text { mandibular } \\
\text { molar }\end{array}$ & $\begin{array}{l}\text { Vital or } \\
\text { nonvital }\end{array}$ \\
\hline
\end{tabular}




\begin{tabular}{|c|c|c|c|c|c|c|c|c|}
\hline & & & & & & $\begin{array}{c}\text { manufactures' } \\
\text { instructions }\end{array}$ & & \\
\hline $\begin{array}{c}\text { Zhou et al. } \\
2012\end{array}$ & $\begin{array}{c}\text { Shanghai } \\
\mathrm{J} \\
\text { Estoma } \\
\text { tol }\end{array}$ & 16 & $\begin{array}{l}\text { Parallel } \\
\text { [Universit } \\
\text { y] }\end{array}$ & $\begin{array}{c}\text { n.r. } \pm \text { n.r. } \\
{[35-} \\
49]\end{array}$ & n.r. $[98] / 0$ & $\begin{array}{c}\text { MANUAL K- } \\
\text { FILES }^{\mathrm{k}}[49] / \# 15 \\
\text { up to \#40 } \\
\text { MTWO }^{\mathrm{c}}[49] / \\
\# 10 / 0.04- \\
\# 15 / 0.05- \\
\# 20 / 0.06- \\
\# 25 / 0.06\end{array}$ & $\begin{array}{c}\text { Mandibular } \\
\text { molar }\end{array}$ & Nonvital \\
\hline
\end{tabular}

ID - identification; SD - standard deviation; yrs - years; \# - number; n.r. - not reported; VAS (Visual Analog Scale): a 10-cm horizontal line with words "no pain" at one end and "worst pain" at the opposite end; NRS (Numerical Rating Scale): none, mild, moderate, considerate, severe.

a Neolix, Châtres-la-Forêt, France. $\quad{ }^{\text {b }}$ FKG dentaire SA, La Chaux-de-Fonds, Switzerland. ${ }^{\mathrm{c}}$ Sweden and Martina, Padua, Italy.

${ }^{\mathrm{d}}$ Dentsply Maillefer, Ballaigues, Switzerland. ${ }^{\mathrm{e}}$ VDW, Munich, Germany. $\quad{ }^{\mathrm{f}}$ Tulsa Dental Products, Tulsa, OK. g SybronEndo, Glendora, CA, USA. ${ }^{\mathrm{h}}$ Kerr Endodontics, Orange, CA, USA. ${ }^{\mathrm{i}}$ Micro-Méga, Besançon Cedex, France. ${ }^{\mathrm{j}}$ ReDent Nova, Ranaana, Israel. ${ }^{k}$ Mani, Tochigi, Japan. ${ }^{1}$ Lightspeed Inc., San Antonio, TX, USA. Source: Authors.

In relation to study design, most studies were parallel, and they were performed at university settings. The mean age reported was 36.1 years (Arslan et al. 2016, Gathani et al. 2016, Jain et al. 2016, Neves et al. 2016, Aqrabawi \& Jamani 2006, Çiçec et al. 2017, Dourado et al. 2005, Gomes et al. 2017, Kashefinejad et al. 2016, Kherlakian et al. 2016, Mollashahi et al. 2017, Neelakantan et al. 2015, Peters et al. 2004, Relvas et al. 2016, Shokraneh et al. 2017, Topcuoglu et al. 2017, Wei et al. 2006); and female patients prevailed in 13 studies (Comparin et al. 2017, Neves et al. 2016, Pasqualini et al. 2016, Aqrabawi \& Jamani 2006, Dourado et al. 2005, Gomes et al. 2017, Dourado et al. 2005, Gomes et al. 2017, Peters et al. 2004 , Wei et al. 2006, Aminsobhani et al. 2017, Krithikadatta et al. 2016, Saumya-Rajesh et al. 2017).

Different instruments were used to perform the endodontic treatment, such as manual K-files, rotatory instruments (Race [Biodental], Mtwo [VDW], Protaper [Maillefer - Dentsply], Profile [Maillefer - Dentsply], Hero [Micro Mega - Injecta], One Shape [Micro Mega - Injecta], Protaper Next [Maillefer - Dentsply], Biorace [Biodental], GT [Maillefer - Dentsply], K3xf [Sybron Endo]), reciprocant instruments (Reciproc [VDW], Wave One [Maillefer - Dentsply], Wave One Gold [Maillefer Dentsply]) and self-adjusting file [ReDentNova]. Various protocols were used in the studies.

The predominant teeth treated was molar (Arslan et al. 2016, Gathani et al. 2016, Jain et al. 2016, Nekoofar et al. 2015, Aqrabawi \& Jamani 2006, Gomes et al. 2017, Kherlakian et al. 2016, Neelakantan et al. 2015, Relvas et al. 2016, Shokraneh et al. 2017, Wei et al. 2006, Aminsobhani et al. 2017, Krithikadatta et al. 2016, Saumya-Rajesh et al. 2017, Dalton et al. 1998, Gambarini et al. 2017, Pettiette et al. 2001, Saha et al. 2018, Shahi et al. 2016, Wang et al. 2010, Zhou et al. 2013). The pulp condition varied between studies, whether it was vital or not. 
Table 4. Summary of the studies selected for this systematic review.

\begin{tabular}{|c|c|c|c|c|c|c|c|}
\hline \multirow[b]{2}{*}{ Study ID } & \multirow[b]{2}{*}{$\begin{array}{c}\text { Anesthesia Salt } \\
{[\mathrm{mL}]}\end{array}$} & \multirow{2}{*}{$\begin{array}{l}\text { \# of } \\
\text { session } \\
\text { s }\end{array}$} & \multirow{2}{*}{$\begin{array}{c}\text { Intracanal } \\
\text { medication/Irri } \\
\text { gation solution }\end{array}$} & \multirow{2}{*}{$\begin{array}{l}\text { Obturation } \\
\text { technique/End } \\
\text { odontic cement }\end{array}$} & \multicolumn{3}{|c|}{ Outcomes } \\
\hline & & & & & $\begin{array}{l}\text { Outcomes } \\
\text { evaluated }\end{array}$ & $\begin{array}{c}\text { evaluat } \\
\text { ion } \\
\text { criteria }\end{array}$ & $\begin{array}{c}\text { Assessment time of } \\
\text { pain }\end{array}$ \\
\hline $\begin{array}{c}\text { Aminsobhani et } \\
\text { al. } 2017\end{array}$ & $\begin{array}{c}\text { 2\% lidocaine } \\
\text { 1:80.000 } \\
\text { epinephrine / } \\
\text { n.r. }\end{array}$ & 1 & $\begin{array}{c}\text {.a. } / 5.25 \% \mathrm{NaOCl} \\
+17 \% \text { EDTA }\end{array}$ & $\begin{array}{c}\text { Lateral } \\
\text { compaction/AH } \\
26\end{array}$ & $\begin{array}{c}\text { Risk, intensity of } \\
\text { pain and } \\
\text { analgesic } \\
\text { consumption }\end{array}$ & NRS 0-3 & $\begin{array}{r}6,12,18 \text { and } 24 \mathrm{~h} \\
\text { after treatment }\end{array}$ \\
\hline $\begin{array}{l}\text { Aqrabawi \& } \\
\text { Jamani } 2006\end{array}$ & $\begin{array}{c}\text { 2\% lidocaine } \\
\text { 1:100.000 } \\
\text { epinephrine/ n.r. }\end{array}$ & 1 & n.a. $/ 2.5 \% \mathrm{NaOCl}$ & $\begin{array}{l}\text { Vertical } \\
\text { compaction/n.r. }\end{array}$ & $\begin{array}{c}\text { Risk and intensity } \\
\text { of pain }\end{array}$ & NRS 0-3 & $\begin{array}{c}8,24 \text { and } 48 \mathrm{~h} \text { after } \\
\text { treatment }\end{array}$ \\
\hline Arias et al. 2015 & $\begin{array}{c}\text { 2\% lidocaine } \\
\text { 1:80.000 } \\
\text { epinephrine / } \\
3.6\end{array}$ & 1 & $\begin{array}{c}\text {.a. } / 5.25 \% \mathrm{NaOCl} \\
+17 \% \text { EDTA }\end{array}$ & $\begin{array}{c}\text { Lateral } \\
\text { compaction/AH } \\
\text { Plus }\end{array}$ & $\begin{array}{r}\text { Risk, intensity and } \\
\text { duration of pain }\end{array}$ & NRS 0-3 & $\begin{array}{c}\text { Every day for } 3 \\
\text { weeks after } \\
\text { treatment }\end{array}$ \\
\hline Arslan et al. 2016 & $\begin{array}{c}\text { 4\% articaine } \\
1: 100.000 \\
\text { epinephrine / } \\
1.7\end{array}$ & 1 & $\begin{array}{c}\text { t.a./1.25\% NaOCl } \\
+17 \% \text { EDTA }\end{array}$ & $\begin{array}{c}\text { Single cone/AH } \\
\text { Plus }\end{array}$ & Intensity of pain & $\begin{array}{c}\text { VAS } 0- \\
100\end{array}$ & $\begin{array}{r}1,3,5 \text { and } 7 \text { days } \\
\text { after treatment }\end{array}$ \\
\hline Çiçec 2017 & n.r. / n.r. & 1 & $\begin{array}{c}\text { n.a. } / 5 \% \mathrm{NaOCl}+ \\
\text { saline }\end{array}$ & $\begin{array}{c}\text { Lateral } \\
\text { compaction/AH } \\
26\end{array}$ & $\begin{array}{c}\text { Risk, intensity of } \\
\text { pain and } \\
\text { unscheluded } \\
\text { appointments } \\
\text { for } \\
\text { complications } \\
\text { emergency }\end{array}$ & NRS 0-4 & $\begin{array}{c}12,24 \text { and } 48 \text { after } \\
\text { treatment }\end{array}$ \\
\hline $\begin{array}{c}\text { Comparin et al. } \\
2017\end{array}$ & $\begin{array}{c}\text { 2\% lidocaine } \\
1: 100.000 \\
\text { epinephrine/ n.r. }\end{array}$ & 1 & $\begin{array}{c}\text { n.a./2.5\% NaOCl } \\
+17 \% \text { EDTA }\end{array}$ & $\begin{array}{l}\text { Continuous } \\
\text { wave/AH Plus }\end{array}$ & $\begin{array}{c}\text { Risk and intensity } \\
\text { of pain }\end{array}$ & NRS 0-3 & $\begin{array}{c}\text { 24, } 48 \text { and } 72 \mathrm{~h} \text { after } \\
\text { treatment }\end{array}$ \\
\hline Dalton et al. 1998 & n.r. / n.r. & n.r. & $\begin{array}{l}\text { Calcium } \\
\text { hydroxide/1\% } \\
\mathrm{NaOCl}+\text { saline }\end{array}$ & n.r./n.r. & $\begin{array}{l}\text { Bacterial } \\
\text { reduction }\end{array}$ & n.a. & n.a. \\
\hline $\begin{array}{l}\text { Dourado et al. } \\
2005\end{array}$ & n.r. / n.r. & 2 & $\begin{array}{c}\text { Calcium } \\
\text { hydroxide/5\% } \\
\mathrm{NaOCl}\end{array}$ & n.r./n.r. & $\begin{array}{l}\text { Bacterial } \\
\text { reduction }\end{array}$ & n.a. & n.a. \\
\hline Endo et al. 2014 & n.r. / n.r. & 1 & $\begin{array}{c}\text { n.a. } / 2 \% \\
\text { chlorexidine gel } \\
+ \text { saline }\end{array}$ & $\begin{array}{c}\text { Lateral } \\
\text { compaction/ } \\
\text { Endomethasone N }\end{array}$ & $\begin{array}{l}\text { Bacterial } \\
\text { reduction }\end{array}$ & n.a. & n.a. \\
\hline Fava 1995 & n.r. / n.r. & 1 & n.a. $/ 0.5 \% \mathrm{NaOCl}$ & $\begin{array}{c}\text { Lateral } \\
\text { compaction/Sea } \\
\text { lapex }\end{array}$ & $\begin{array}{c}\text { Risk and intensity } \\
\text { of pain }\end{array}$ & NRS 0-3 & $\begin{array}{l}48 \mathrm{~h} \text { and } 1 \text { week } \\
\text { after treatment }\end{array}$ \\
\hline $\begin{array}{c}\text { Gambarini et al. } \\
2017\end{array}$ & n.r. / n.r. & 1 & n.a. $/ 5 \% \mathrm{NaOCl}$ & n.r./n.r. & $\begin{array}{c}\text { Risk and intensity } \\
\text { of pain }\end{array}$ & NRS 0-3 & $\begin{array}{c}\text { For } 3 \text { days after } \\
\text { treatment }\end{array}$ \\
\hline $\begin{array}{l}\text { Gatthani \& } \\
\text { Raghavendra } \\
2016\end{array}$ & n.r. / n.r. & 1 or 2 & $\begin{array}{c}\text { n.a. or calcium } \\
\text { hydroxide/3\% } \\
\mathrm{NaOCl}\end{array}$ & $\begin{array}{c}\text { Lateral } \\
\text { compaction/RC } \\
\text { fill }\end{array}$ & Intensity of pain & $\begin{array}{c}\text { VAS } 0- \\
100\end{array}$ & $\begin{array}{c}1,3 \text { and } 7 \text { days after } \\
\text { treatment }\end{array}$ \\
\hline Gomes et al. 2017 & $2 \%$ lidocaine & 1 & n.a. $/ 2 \%$ & Continuous wave & Risk and intensity & VAS 0- & Immediately \\
\hline
\end{tabular}


Research, Society and Development, v. 10, n. 8, e41910817350, 2021

(CC BY 4.0) | ISSN 2525-3409 | DOI: http://dx.doi.org/10.33448/rsd-v10i8.17350

\begin{tabular}{|c|c|c|c|c|c|c|c|}
\hline & $\begin{array}{c}\text { 1:100.000 } \\
\text { epinephrine/ n.r. }\end{array}$ & & $\begin{array}{l}\text { chlorexidine gel } \\
+ \text { saline }\end{array}$ & $\begin{array}{c}\text { and vertical } \\
\text { compation/Endo } \\
\text { methone-N }\end{array}$ & of pain & 100 & \\
\hline Jain et al. 2016 & $\begin{array}{c}\text { 2\% lidocaine } \\
\text { 1:100.000 } \\
\text { epinephrine/ n.r. }\end{array}$ & 1 & n.a. $/ 2.5 \% \mathrm{NaOCl}$ & $\begin{array}{l}\text { Continuous } \\
\text { wave/AH Plus }\end{array}$ & $\begin{array}{c}\text { Risk, intensity of } \\
\text { pain and } \\
\text { analgesic } \\
\text { consumption }\end{array}$ & $\begin{array}{c}\text { unctiona } \\
1 \text { pain } \\
\text { scale }\end{array}$ & $\begin{array}{c}24,48,72 \text { and } 7 \\
\text { days after } \\
\text { treatment }\end{array}$ \\
\hline $\begin{array}{c}\text { Kashofinejad et } \\
\text { al. } 2016\end{array}$ & $\begin{array}{l}\text { 2\% lidocaine } \\
\text { 1:80.000 } \\
\text { epinephrine/ } 1.5\end{array}$ & 1 & n.a./Saline & $\begin{array}{c}\text { Lateral } \\
\text { compaction/AH } \\
26\end{array}$ & $\begin{array}{c}\text { Risk and intensity } \\
\text { of pain }\end{array}$ & $\begin{array}{r}\text { VAS 0- } \\
100\end{array}$ & $\begin{array}{r}4,8,12 \text { and } 24 \mathrm{~h} \\
\text { after treatment }\end{array}$ \\
\hline $\begin{array}{c}\text { Kherlakian et al. } \\
2016\end{array}$ & $\begin{array}{c}\text { 2\% lidocaine } \\
\text { 1:100.000 } \\
\text { epinephrine/ n.r. }\end{array}$ & 1 & n.a. $/ 2.5 \% \mathrm{NaOCl}$ & $\begin{array}{l}\text { Continuous } \\
\text { wave/AH Plus }\end{array}$ & $\begin{array}{c}\text { Intensity of pain } \\
\text { and analgesic } \\
\text { consuption }\end{array}$ & NRS 0-3 & $\begin{array}{c}24,48,72 \text { and } 1 \\
\text { week after } \\
\text { treatment }\end{array}$ \\
\hline $\begin{array}{l}\text { krithikadatta et al. } \\
2016\end{array}$ & $\begin{array}{c}\text { 2\% lidocaine } \\
\text { 1:80.000 } \\
\text { epinephrine/ n.r. }\end{array}$ & n.r. & n.a. $/ 5 \% \mathrm{NaOCl}$ & n.r./n.r. & Intensity of pain & $\begin{array}{r}\text { VAS 0- } \\
100\end{array}$ & $\begin{array}{c}2,4,6,12,24,36 \\
\text { and } 48 \mathrm{~h} \text { after } \\
\text { treatment }\end{array}$ \\
\hline $\begin{array}{c}\text { Mollashahi et al. } \\
2017\end{array}$ & $\begin{array}{l}\text { 2\% lidocaine } \\
\text { 1:80.000 } \\
\text { epinephrine/ n.r. }\end{array}$ & 1 & n.a. $/ 2.5 \% \mathrm{NaOCl}$ & $\begin{array}{c}\text { Lateral } \\
\text { compaction/AH } \\
26\end{array}$ & Intensity of pain & $\begin{array}{r}\text { VAS 0- } \\
170\end{array}$ & $\begin{array}{r}6,12,24,48 \text { and } 72 \\
\text { h after treatment }\end{array}$ \\
\hline $\begin{array}{c}\text { Nekoofar et al. } \\
2015\end{array}$ & $\begin{array}{c}\text { 2\% lidocaine } \\
\text { 1:80.000 } \\
\text { epinephrine/ n.r. }\end{array}$ & 2 & $\begin{array}{l}\text { None } / 2 \% \\
\text { chlorexidine }\end{array}$ & $\begin{array}{c}\text { Lateral } \\
\text { compaction/AH } \\
26\end{array}$ & $\begin{array}{c}\text { Intensity of pain, } \\
\text { canal } \\
\text { preparation time } \\
\text { and analgesic } \\
\text { consuption }\end{array}$ & $\begin{array}{r}\text { VAS 0- } \\
100\end{array}$ & $\begin{array}{c}6,12,18,24,48 \text { and } \\
72 \mathrm{~h} \text { after } \\
\text { treatment }\end{array}$ \\
\hline $\begin{array}{r}\text { Neelakantan \& } \\
\text { Sharma } 2015\end{array}$ & $\begin{array}{c}\text { 2\% lidocaine } \\
\text { 1:80.000 } \\
\text { epinephrine/ n.r. }\end{array}$ & 1 & n.a./3\% $\mathrm{NaOCl}$ & $\begin{array}{c}\text { Vertical } \\
\text { compaction/MT } \\
\text { A Plus }\end{array}$ & $\begin{array}{c}\text { Risk and intensity } \\
\text { of pain }\end{array}$ & NRS 0-3 & $\begin{array}{c}\text { For } 7 \text { days after } \\
\text { treatment }\end{array}$ \\
\hline Neves et al. 2016 & n.r. / n.r. & 2 & $\begin{array}{c}\text { Calcium } \\
\text { hydroxide } / 2.5 \% \\
\mathrm{NaOCl}\end{array}$ & $\begin{array}{l}\text { Lateral } \\
\text { compaction/n.r. }\end{array}$ & $\begin{array}{l}\text { Bacterial } \\
\text { reduction }\end{array}$ & n.a. & n.a. \\
\hline $\begin{array}{c}\text { Pasqualini et al. } \\
2016\end{array}$ & n.r. / n.r. & 1 & n.a. $/ 5 \% \mathrm{NaOCl}$ & $\begin{array}{c}\text { Continuous } \\
\text { wave/Pulp canal } \\
\text { sealer }\end{array}$ & Intensity of pain & NRS 0-4 & $\begin{array}{c}\text { Each day for } 7 \text { days } \\
\text { after treatment }\end{array}$ \\
\hline Peters et al. 2004 & n.r. / n.r. & 1 or 2 & $\begin{array}{c}\text { Calcium } \\
\text { hydroxide } / 2.5 \% \\
\mathrm{NaOCl}+17 \% \\
\text { EDTA }\end{array}$ & $\begin{array}{c}\text { Lateral } \\
\text { compaction or } \\
\text { Continuous } \\
\text { wave/AH Plus }\end{array}$ & $\begin{array}{c}\text { Success of } \\
\text { endodontic } \\
\text { treatment }\end{array}$ & n.a. & n.a. \\
\hline $\begin{array}{l}\text { Pettiette et al. } \\
\qquad 2001\end{array}$ & n.r. / n.r. & 2 & n.r./n.r. & n.r./n.r. & $\begin{array}{c}\text { Success of } \\
\text { endodontic } \\
\text { treatment }\end{array}$ & n.a. & n.a. \\
\hline Relvas et al. 2016 & $\begin{array}{c}2 \% \text { lidocaine } \\
\text { 1:100.000 } \\
\text { epinephrine / } \\
3.6\end{array}$ & 1 & $\begin{array}{c}\text { n.a. } / 2.5 \% \mathrm{NaOCl} \\
+ \text { saline }\end{array}$ & $\begin{array}{l}\text { Single cone }+ \\
\text { thermomechani } \\
\text { cal } \\
\text { compaction/AH } \\
\text { Plus }\end{array}$ & $\begin{array}{c}\text { Risk and intensity } \\
\text { of pain }\end{array}$ & $\begin{array}{r}\text { VAS 0- } \\
100\end{array}$ & $\begin{array}{c}24,72 \mathrm{~h} \text { and } 1 \text { week } \\
\text { after treatment }\end{array}$ \\
\hline Saha et al. 2018 & $\begin{array}{c}\text { 2\% lidocaine } \\
\text { 1:80.000 } \\
\text { epinephrine / } \\
\text { n.r. }\end{array}$ & 1 & ..a. $/ 5.25 \% \mathrm{NaOCl}$ & $\begin{array}{c}\text { Lateral } \\
\text { compaction/AH } \\
\text { Plus }\end{array}$ & Intensity of pain & $\begin{array}{c}\text { VAS 0- } \\
100\end{array}$ & $\begin{array}{c}24,48,72 \mathrm{~h} \text { and } 1 \\
\text { week after } \\
\text { treatment }\end{array}$ \\
\hline Saumya-Rajesh et & $2 \%$ lidocaine & 2 & Calcium & n.r./n.r. & Intensity of pain & VAS 0- & $2,4,6,8,24$ and 48 \\
\hline
\end{tabular}




\begin{tabular}{|c|c|c|c|c|c|c|c|}
\hline al. 2017 & $\begin{array}{c}\text { 1:80.000 } \\
\text { epinephrine / } \\
\text { n.r. }\end{array}$ & & $\begin{array}{c}\text { hydroxide/3\% } \\
\mathrm{NaOCl}\end{array}$ & & & 100 & $\mathrm{~h}$ after treatment \\
\hline Shahi et al. 2016 & $\begin{array}{c}\text { 2\% lidocaine } \\
\text { 1:80.000 } \\
\text { epinephrine / } \\
\text { n.r. }\end{array}$ & 1 & $\begin{array}{c}\text { n.a. } / 2.5 \% \mathrm{NaOCl} \\
+17 \% \mathrm{EDTA} \\
\text { gel-form }\end{array}$ & $\begin{array}{c}\text { Lateral } \\
\text { compaction/AH } \\
26\end{array}$ & $\begin{array}{c}\text { Risk and intensity } \\
\text { of pain }\end{array}$ & NRS 0-3 & $\begin{array}{c}4,8,12,24,48,72 \mathrm{~h} \\
\text { and } 1 \text { week after } \\
\text { treatment }\end{array}$ \\
\hline $\begin{array}{c}\text { Shokraneh et al. } \\
2016\end{array}$ & $\begin{array}{c}2 \% \text { lidocaine } \\
1: 80.000 \\
\text { epinephrine / } \\
1.8\end{array}$ & 1 & ..а. $/ 5.25 \% \mathrm{NaOCl}$ & $\begin{array}{c}\text { Lateral } \\
\text { compaction/AH } \\
26\end{array}$ & $\begin{array}{c}\text { Risk and intensity } \\
\text { of pain }\end{array}$ & $\begin{array}{c}\text { VAS 0- } \\
100\end{array}$ & $\begin{array}{c}6,12,18,24,48 \text { and } \\
72 \mathrm{~h} \text { after } \\
\text { treatment }\end{array}$ \\
\hline $\begin{array}{l}\text { Topçuaglu \& } \\
\text { Topçuaglu } 2017\end{array}$ & $\begin{array}{c}\text { 4\% articaine } \\
\text { 1:200.000 } \\
\text { epinephrine / } \\
\text { n.r. }\end{array}$ & 2 & $\begin{array}{c}\text { Calcium } \\
\text { hydroxide } / 2.5 \% \\
\mathrm{NaOCl}\end{array}$ & $\begin{array}{l}\text { Lateral } \\
\text { compaction/ } \\
\text { MM seal }\end{array}$ & $\begin{array}{c}\text { Risk, intensity of } \\
\text { pain and time to } \\
\text { remove canal } \\
\text { filling }\end{array}$ & NRS $0-3$ & $\begin{array}{c}6,12,24,48,72 \mathrm{~h}, 7 \\
\text { and } 10 \text { days after } \\
\text { treatment }\end{array}$ \\
\hline Wang et al. 2010 & n.r. / n.r. & n.r. & $\begin{array}{c}\text { n.r. } / 1 \% \mathrm{NaOCl}+ \\
3 \% \mathrm{H}_{2} \mathrm{OH}\end{array}$ & $\begin{array}{c}\text { Lateral } \\
\text { compaction/AH } \\
\text { Plus }\end{array}$ & $\begin{array}{c}\text { Risk and intensity } \\
\text { of pain }\end{array}$ & $\begin{array}{l}\text { Heft- } \\
\text { Parker } \\
0-170\end{array}$ & n.r. \\
\hline Wey et al. 2003 & n.r. / n.r. & 2 & $\begin{array}{l}\text { Calcium } \\
\text { hydroxide/n.r. }\end{array}$ & n.r./n.r. & $\begin{array}{c}\text { Risk and intensity } \\
\text { of pain }\end{array}$ & NRS 0-3 & $\begin{array}{c}7 \text { and } 10 \text { days after } \\
\text { treatment }\end{array}$ \\
\hline Zhou et al. 2012 & n.r. / n.r. & 1 & n.a. $/ 1 \% \mathrm{NaOCl}$ & $\begin{array}{c}\text { Lateral } \\
\text { compaction/AH } \\
\text { Plus }\end{array}$ & $\begin{array}{c}\text { Risk of pain and } \\
\text { success of } \\
\text { endodontic } \\
\text { treatment }\end{array}$ & n.r. & $\begin{array}{c}7 \text { days after } \\
\text { endodontic } \\
\text { treatment }\end{array}$ \\
\hline
\end{tabular}

ID - identification; \# - number; n.r. - not reported; n.a. - not applied. Source: Authors.

The 2\% lidocaine was the anesthetic salt most used in the studies (Comparin et al. 2017, Jain et al. 2016, Nekoofar et al. 2015, Aqrabawi \& Jamani 2006, Gomes et al. 2017, Kashefinejad et al. 2016, Kherlakian et al. 2016, Mollashahi et al. 2017, Neelakantan et al. 2015, Relvas et al. 2016, Shokraneh et al. 2017, Aminsobhani et al. 2017, Krithikadatta et al. 2016, Saumya-Rajesh et al. 2017, Saha et al. 2018, Shahi et al. 2016, Arias et al. 2015). Only 9 studies out of 33 performed the endodontic treatment in 2 sessions (Gathani et al. 2016, Nekoofar et al. 2015, Neves et al. 2016, Dourado et al. 2005, Peters et al. 2004, Topcuoglu et al. 2017, Wei et al. 2006, Saumya-Rajesh et al. 2017, Pettiette et al. 2001), and most of them used calcium hydroxide as intracanal medication.

In relation to irrigant solution, sodium hypochlorite in different concentrations, was the most often used. Different obturation techniques were used, such as lateral compaction, vertical compaction, single cone, and continuous wave. A resinbased type endodontic cement was the most used one (AH Plus - Dentsply Sirona, York, Pennsylvania, USA).

Different outcomes were evaluated in the primary studies: risk, intensity and duration of pain, analgesic consumption, bacterial reduction, and success of endodontic treatment.

\section{Risk of bias within studies}

In relation to the overall risk of bias, only eight studies out of 33 were classified at low risk of bias, representing $24 \%$ of the studies evaluated (Çiçec et al. 2017, Gomes et al. 2017, Mollashahi et al. 2017, Relvas et al. 2016, Shokraneh et al. 2017, Aminsobhani et al. 2017, Krithikadatta et al. 2016, Pasqualini et al. 2015). Seven were classified at high risk of bias (Nekoofar et al. 2015, Aqrabawi \& Jamani 2006, Gambarini et al. 2017, Pettiette et al. 2001, Zhou et al. 2013, Fava 1995, Wang et al. 2010) and 18 studies were classified at some concerns (Arslan et al. 2016, Comparin et al. 2017, Gathani et al. 2016, Jain et al. 2016, Neves et al. 2016, Dourado et al. 2005, Kherlakian et al. 2016, Neelakantan et al. 2015, Peters et al. 
2004, Topcuoglu et al. 2017, Wei et al. 2006, Saumya-Rajesh et al. 2017, Dalton et al. 1998, Saha et al. 2018, Shahi et al. 2016, Arias et al. 2015, Endo et al 2014) (Figure 2).

Figure 2. Summary of the risk of bias assessment according to the RoB 2.0 (Cochrane Collaboration tool).

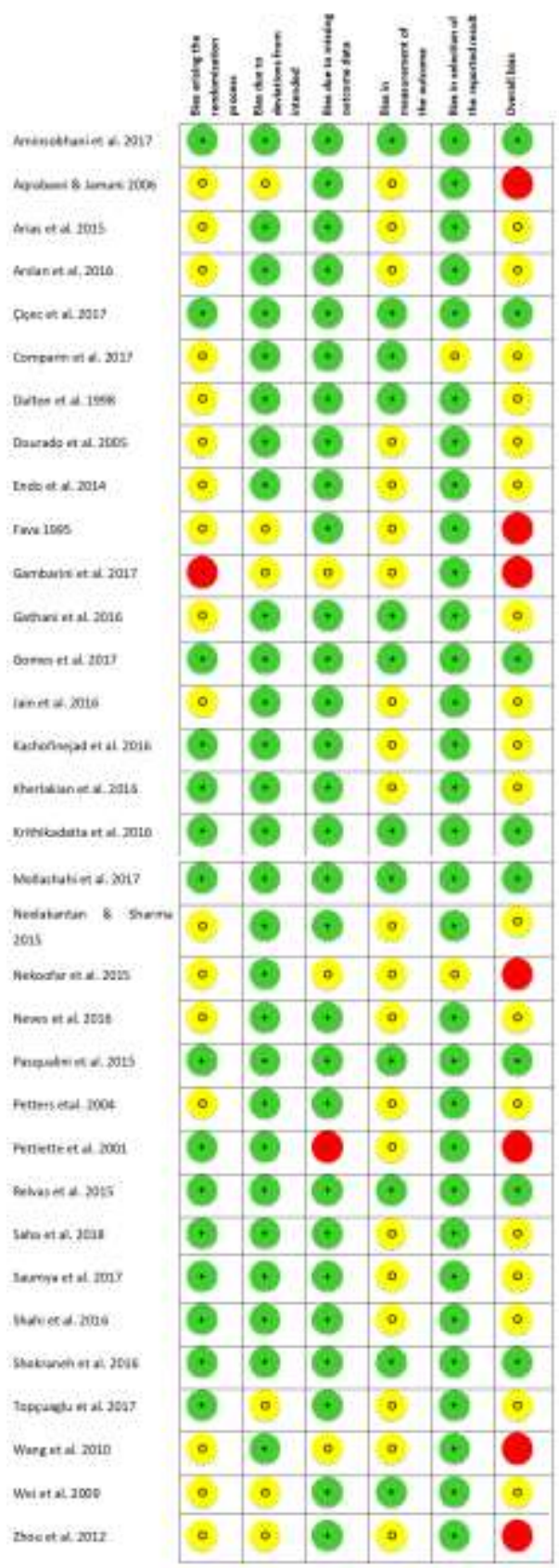

Source: Authors.

\section{Study compliance with each of the CONSORT instrument tool items}

The overall CONSORT score for the studies included in this review was $22.2 \pm 6.2$ points, which represents $69 \%$ of the maximum CONSORT score of 32 points. Figure 3 shows the percentage of studies per CONSORT score for each CONSORT item evaluated. 
Research, Society and Development, v. 10, n. 8, e41910817350, 2021

(CC BY 4.0) | ISSN 2525-3409 | DOI: http://dx.doi.org/10.33448/rsd-v10i8.17350

Figure 3. Percentage of studies per CONSORT score for each CONSORT item evaluated.

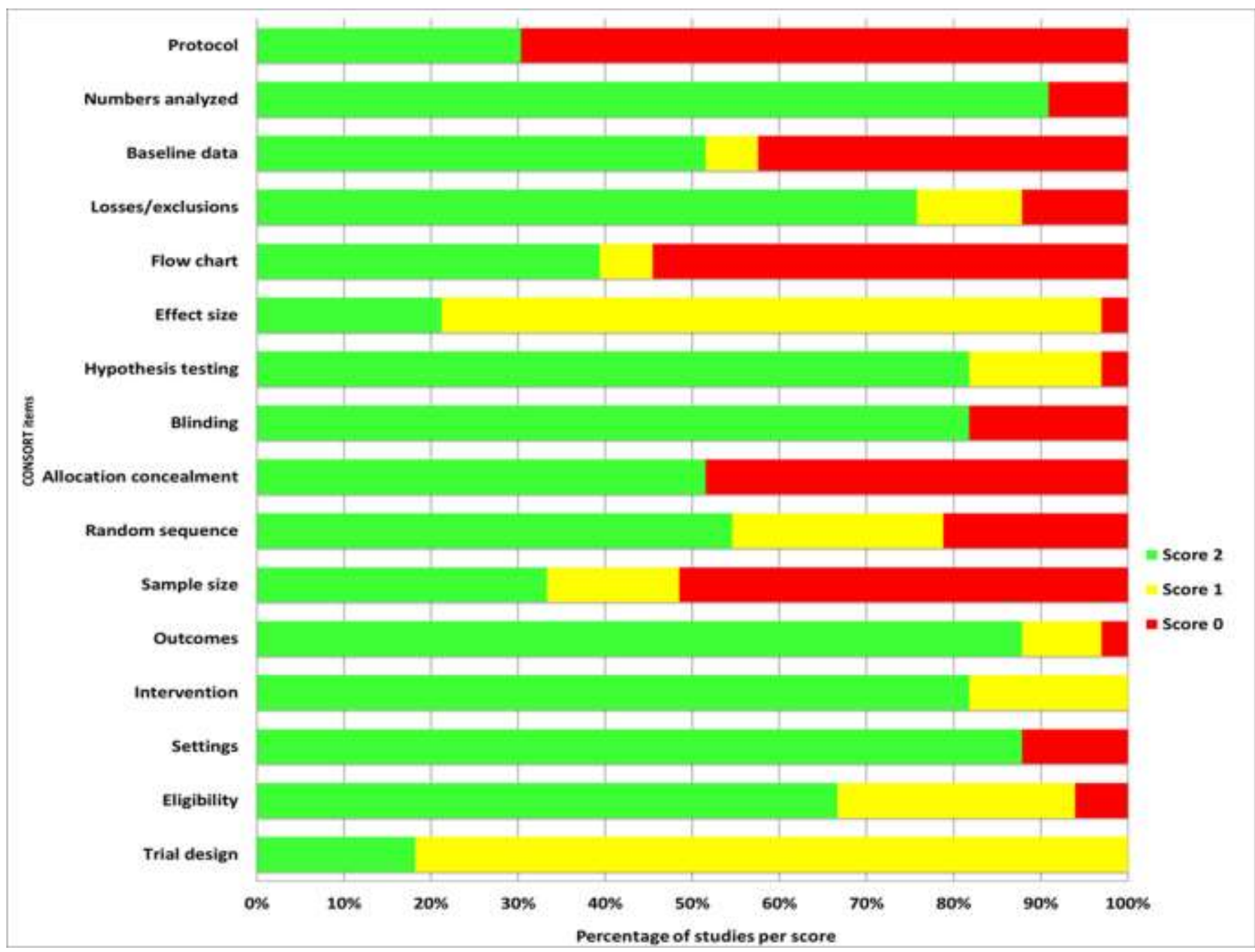

Source: Authors.

The items classified as score 0 or 1 in most studies were: protocol, flow chart, effect size, sample size, and trial design. Random sequence had a high percentage of scores 0 and 1 , while allocation concealment received mostly score 0.

The items scored as 2 in most of the studies were the following: numbers analyzed, baseline data, losses/exclusions, hypothesis testing, blinding, outcomes, interventions, settings, and eligibility. 
Research, Society and Development, v. 10, n. 8, e41910817350, 2021

(CC BY 4.0) | ISSN 2525-3409 | DOI: http://dx.doi.org/10.33448/rsd-v10i8.17350

Figure 4. Dispersion chart showing the weak correlation between the journal impact factor and the overall CONSORT score.

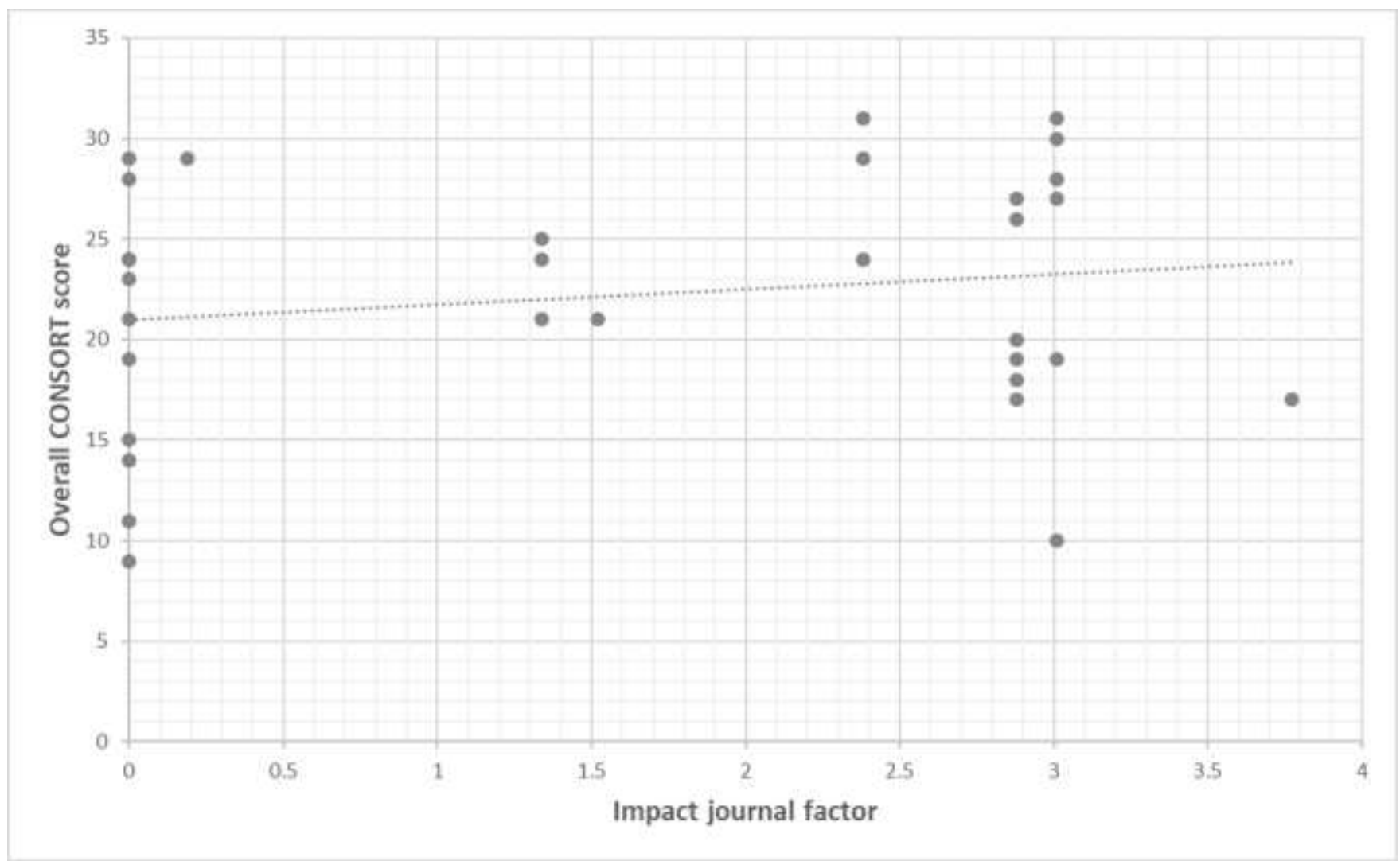

Source: Authors.

Correlation between journal impact factor and overall CONSORT score $(r=0.16 ; p=0.359$; Figure 4) was week and not significant, representing only $3 \%$ of the dispersion of data $(\mathrm{R} 2=0.03$, Figure 4$)$. 
Table 5. Average CONSORT score per journal, country and period.

\begin{tabular}{|c|c|c|c|c|c|}
\hline Variables & Categories & $\begin{array}{c}\text { Number of } \\
\text { studies } \\
\text { (n = } 33 \text { total) }\end{array}$ & Mean \pm SD & Median (interquartile range) & p-value* \\
\hline \multirow{8}{*}{ Journal } & J Endod & 6 & $21.2 \pm 4.3$ & $19.5(18-25)$ & \multirow{8}{*}{0.36} \\
\hline & Int Endod $\mathrm{J}$ & 6 & $24.2 \pm 8.2$ & $27.5(21-29)$ & \\
\hline & J Appl Oral Sci & 3 & $23.4 \pm 2.1$ & $24(21-25)$ & \\
\hline & Clin Oral Investig & 3 & $28.0 \pm 3.6$ & $29(24-31)$ & \\
\hline & J Conserv Dent & 2 & $26.5 \pm 3.5$ & $26.5(24-29)$ & \\
\hline & Shanghai J Stomatol & 2 & $15.0 \pm 1.4$ & $15(14-16)$ & \\
\hline & Endo & 2 & $22.0 \pm 1.4$ & $22(21-23)$ & \\
\hline & Others & 9 & $19.2 \pm 7.0$ & $19(15-24)$ & \\
\hline \multirow{8}{*}{ Country } & Brazil & 8 & $22.8 \pm 6.9$ & $23.5(19-28)$ & \multirow{8}{*}{0.03} \\
\hline & China & 3 & $15.0 \pm 1.0$ & $15(14-16)$ & \\
\hline & India & 6 & $25.3 \pm 3.8$ & $24(23-28)$ & \\
\hline & Iran & 6 & $26.0 \pm 3.4$ & $27.5(25-28)$ & \\
\hline & Italy & 2 & $19.5 \pm 14.8$ & $19.5(9-30)$ & \\
\hline & USA & 2 & $17.5 \pm 0.7$ & $17.5(17-18)$ & \\
\hline & Turkey & 3 & $23.3 \pm 2.1$ & $24(21-25)$ & \\
\hline & Others & 3 & $15.7 \pm 4.2$ & $17(11-19)$ & \\
\hline \multirow{4}{*}{ Period } & $1995-2000$ & 2 & $14.0 \pm 5.7$ & $14(10-18)$ & \multirow{4}{*}{0.01} \\
\hline & $2000-2005$ & 4 & $18.0 \pm 2.6$ & $18(17-20)$ & \\
\hline & $2006-2010$ & 2 & $12.5 \pm 2.1$ & $12.5(11-14)$ & \\
\hline & 2011-2018 & 25 & $24.0 \pm 5.7$ & $25(20-28)$ & \\
\hline \multirow{3}{*}{ RoB } & Low & 8 & $28.6 \pm 1.8$ & $29(28-29.5)$ & \multirow{3}{*}{$<0.001$} \\
\hline & Some concerns & 18 & $22.2 \pm 4.1$ & $22(19-24)$ & \\
\hline & High & 7 & $13.9 \pm 4.1$ & $14(10.25-16.75)$ & \\
\hline
\end{tabular}

Source: Authors.

No influence of the journal on the average CONSORT score was observed ( $\mathrm{p}=0.36$; Table 5). On the other hand, significant differences among countries were observed $(\mathrm{p}=0.03$; Table 5).

The vast majority of the RCT comparing endodontic treatments was published from 2011 to 2018 (25 out of 33). The year range had a significant influence on the average CONSORT score, with higher average CONSORT score in the more recent field of years ( $p=0.01$; Table 5$)$.

The RoB of the studies had a significant influence on the average CONSORT score, with higher average CONSORT score in studies classified at 'low risk of bias' $(\mathrm{p}=0.001$; Table 5).

\section{Discussion}

In order to evaluate the adherence of the RCTs with the CONSORT Statement, a scale from 0 to 2 was used, with zero meaning absence of reporting, 1 bad report, and 2 adequate report (Reis et al. 2018). To the best of authors' knowledge, this is the first study that attempted to evaluate the adherence of endodontic instrumentation RCTs to the CONSORT Statement, which was one of the objectives of this study.

A total of 12 items of the CONSORT Statement were included in this CONSORT evaluation tool: trial design, participants, interventions, outcomes, sample size, randomization, blinding, statistical methods, participant flow, baseline data, numbers analyzed and registration and protocol of these items. Those who contributed the most to lowering the CONSORT 
score were random sequence and allocation concealment.

Adequate randomization balances, both known and unknown prognostic factors in the allocation of treatments. In addition to randomization, concealment of allocation is also important as it protects the randomization process so that the treatment to be allocated is not discovered before the patient has participated in the study. Proper management of these two domains minimizes selection bias (Higging et al. 2011). There is evidence that improper conduction of these steps in RCTs increases the likelihood of systematic errors (Pocock 1983); studies with poor methodological quality tend to overestimate the results, favoring the intervention in the test group (Schulz et al. 1995, Khan et al. 1996).

Masking or blinding is the process of retaining information about the interventions assigned to each group out of examiners and patients, and it is a critical element in the design of RCTs. When done successfully, it prevents the introduction of information bias (Feys et al. 2014). Blinding can be applied to participants, researchers, and evaluators of study results. In clinical trials of endodontic instrumentation, masking poses a more significant challenge than for drug therapies. It is impossible to prevent the care provider, i.e., the dentist, from knowing which technique and instruments will be used for endodontic treatment. Therefore, the masking of the operator is not feasible. Evaluators can, and should, preferably, be masked on group assignment.

Compared with other areas of Dentistry or with the number of laboratory studies in Endodontics, there are still few RCTs on endodontic instrumentation. Only 33 studies were identified for inclusion in this systematic review, and only $24 \%$ (8/33) of the studies were classified at low risk of bias in the field, which shows a new need for new well-designed randomized controlled clinical studies.

Ideally, an average CONSORT score of 32 points should be obtained, meaning that the studies adhered entirely to the CONSORT statement. However, these 33 eligible studies on endodontic instrumentation had an overall average CONSORT score of $22.2 \pm 6.2$, which was slightly superior to that observed in bleaching studies (16.7 \pm 5.4 ) (Loguercio et al. 2017) and studies of bonding conducted in non-carious cervical lesions (15.0 \pm 4.8 ) (Reis et al. 2018). This superior average score in the endodontic instrumentation area is probably attributed to the fact that most studies were conducted in the most recent years when authors had a better understanding of the steps required for the conduction of and rigorous and adequate RCTs.

In addition to the overall CONSORT score, comparisons were made related to the journal, country of publication, and period of publication. These additional analyses aimed to provide information on whether improvements in the average CONSORT scores occurred over time and whether these improvements were related to the journal and its impact factor, as well as the country of origin of the first author. No influence of the journal on the average CONSORT score was observed. However, significant differences among the publication period were observed with a higher average CONSORT score in the most recent, in agreement with earlier systematic reviews (Loguercio et al. 2017, Reis et al. 2018).

One should point out the limitations of the studies included in the present study. A systematic review is a collection of the eligible studies and cannot report on findings not addressed by the primary studies. The first limitation of the eligible studies is that they reported surrogate outcomes (risk or intensity of pain, analgesic consumption, and bacterial reduction), instead of true outcomes (complete remission of the disease, absence of periapical lesion, maintenance of the tooth in the mouth or patient's preference; mean survival rate of the teeth). Surrogate endpoints might be problematic on some occasions since they are not always excellent representatives of the real clinical outcome. Unfortunately, however, the use of surrogate outcomes that try to predict the effect of specific treatment on an unobserved exact outcome have substantial economic and ethical advantages, reducing the duration and size of clinical trials (Ensor et al. 2016) they have been used very often in RCTs.

Some essential items such as study flow chart, sample size calculation, method of sample randomization, and allocation concealment should be added to the RCTs' methodology in order to improve the quality of the studies. It is important to be aware of the quality of RCTs in the field of endodontic instrumentation, as RCTs are considered the "gold 
standard" for treatment evaluation and serve as a reference for dentists' decision making. However, its presentation is not always sufficiently clear to allow adequate analysis of the findings and conclusions; therefore, a systematic review evaluating adherence to CONSORT and the risk of bias of RCTs evaluating endodontic instrumentation systems is relevant.

A limitation of this systematic review is that not all articles published in journals are available. To evaluate the CONSORT items, it is necessary to obtain the full text of the articles; this is the reason the gray literature was not explored.

Future studies of endodontic instrumentation should be better delineated, with complete information on the study methodology so that their risk of bias can be evaluated.

\section{Conclusion}

The adherence of RCTs that evaluate endodontic instrumentation to the CONSORT statement requires improvements. Adherence to the CONSORT statement will also reduce the high or some concerns risk of bias of studies in the field.

More well-designed clinical studies evaluating endodontic instrumentation should be carried out in order to have more reliable results.

\section{References}

Altman, D. G., Schulz, K. F., Moherm D., Egger, M., Davidoff, F., Elbourne, D., Gøtzsche, P. C., Lang, T.; CONSORT GROUP (Consolidated Standards of Reporting Trials) (2001) The revised CONSORT statement for reporting randomized trials: explanation and elaboration. Annals of Internal Medicine 134(8), 663-694.

Aminsobhani, M., Meraji, N., Khoshdel, A., \& Ghorbanzadeh, A. (2017) The Effect of Root Canal Preparation Using Single Versus Multiple Endodontic Rotary Files on Post-operative Pain, a Randomised Clinical Trial. European Endodontic Journal 2(1), 1-5.

Aqrabawi, J., \& Jamani, K. (2006) Prevalence of post-treatment pain after cleaning and shaping of the root canal system using manual step-back versus rotary nickel titanium. Odonto-stomatologie Tropicale 29(113), 5-9.

Arias, A., de la Macorra, J. C., Azabal, M., Hidalgo, J. J., \& Peters, O.A. (2015) Prospective case controlled clinical study of post-endodontic pain after rotary root canal preparation performed by a single operator. Journal of Dentistry 43(3), 389-395.

Arslan, H., Khalilov, R., Doanay, E., \& Karatas, E. (2016) The effect of various kinematics on postoperative pain after instrumentation: a prospective, randomized clinical study. Journal of Applied Oral Science 24(5), 503-508.

Bartols, A., Laux, G., \& Walther, W. (2016) Multiple-file vs. single-file endodontics in dental practice: a study in routine care. PeerJ 4:e2765.

Chugal, N., Mallya, S. M., Kahler, B., \& Lin, L. M. (2017) Endodontic Treatment Outcomes. Dental Clinics of North America 61, 59-80.

Çiçek, E., Koçak, M., Koçak, S., Salam, B., \& Türker, S. (2017) Postoperative pain intensity after using different instrumentation techniques: a randomized clinical study. Journal of Applied Oral Science 25(1), 20-26.

Comparin, D., Moreira, E., Souza, E., De-Deus, G., Arias, A., \& Silva, E. (2017) Postoperative Pain after Endodontic Retreatment Using Rotary or Reciprocating Instruments: a Randomized Clinical Trial. Journal of Endodontics 43(7), 1084-1088.

Dalton, B., Orstavik, D., Phillips, C., Pettiette, M., \& Trope, M. (1998) Bacterial reduction with nickel-titanium rotary instrumentation. Journal of Endodontics 24(11), 763-767.

Darda, S., Manwar, N., Chandak, M., \& Shori, D. D. (2009) An in vivo evaluation of two types of files used to accurately determine the diameter of the apical constriction of a root canal: an in vivo study. Journal of Contemporary Dental Practice 10(4), 43-50.

Del Fabbro, M., Afrashtehfar, K. I., Corbella, S., El-Kabbaney, A., Perondi, I., \& Taschieri, S. (2018) In Vivo and In Vitro Effectiveness of Rotary NickelTitanium vs Manual Stainless Steel Instruments for Root Canal Therapy: Systematic Review and Meta-analysis. Journal of Evidence-Based Dental Practice 18(1), 59-69.

Dourado, A. T., Caldas Junior, A. de F., Alves, D. F., \& Falcao, C. A. (2005) Bacteriemia during endodontic treatment in relation to the technique of biomechanical preparation: randomized clinical trial. Journal of Applied Oral Science 13(4), 334-339.

Endo, M. S., Signoretti, F. G. C., Pavan, N. N. O., Martinho, F. C., \& Gomes, B. P. F. A. (2014) Effectiveness of hand iles and the MTwo R system in bacterial reduction in endodontically treated teeth with chronic apical periodontitis. Dental Press Endodontics 4(3), 21-27.

Ensor, H., Lee, R. J., Sudlow, C., \& Weir, C. J. (2016) Statistical approaches for evaluating surrogate outcomes in clinical trials: A systematic review. Journal of Biopharmaceutical Statistics 26(5), 859-879.

Fava, L. R. G. (1995) Single visit root canal treatment: incidence of postoperative pain using three different instrumentation techniques. International Endodontic Journal 28(2), 103-107. 
Feys, F., Bekkering, G. E., Singh, K., \& Devroey, D. (2014) Do randomized clinical trials with inadequate blinding report enhanced placebo effects for intervention groups and nocebo effects for placebo groups? Systematic Review 3, 14.

Gambarini, G., Nardo, D., Miccoli, G., Guerra, F., Di Giorgio, R., Di Giorgio, G., Glassman, G., Piasecki, L., \& Testarelli, L. (2017) The influence of a new clinical motion for endodontic instruments on the incidence of postoperative pain. Clinica Terapeutica 16(8), e23-e27.

Gathani, K., \& Raghavendra, S. S. (2016) Comparison of postoperative pain in single-visit and multiple-visit endodontic therapy using ProTaper Universal, Hero Shaper and the Self-adjusting File - A split-mouth clinical trial. Endo-Endodontic Practice Today 10(1), 23-28.

Gomes, A. C., Soares, A. J., Souza, E. M., Zaia, A. A., \& Silva, E. (2017) Intraoperative discomfort associated with the use of a rotary or reciprocating system: a prospective randomized clinical trial. Restorative Dentistry and Endodontics 42(2), 140-145.

Higgins, J. P., Altman, D. G., Gotzsche, P. C., Jüni, P., Moher, D., Oxman, A. D., Savovic, J., Schulz, K. F., Weeks, L., Sterne, J. A.; Cochrane Bias Methods Group; Cochrane Statistical Methods Group. (2011) The Cochrane Collaboration's tool for assessing risk of bias in randomised trials. BMJ 343 , d5928.

Jain, N., Pawar, A. M., Naganath, M., Gupta, A., \& Daryani, H. (2016) Incidence and severity of postoperative pain after canal instrumentation with reciprocating system, continuous rotary single file system, versus SAF system. Endo-Endodontic Practice Today 10(3), 153-160.

Jardine, A. P., da Rosa, R. A., Santini, M. F., Zaccara, I. M., So, M. V. R., \& Kopper, P. M. P. (2016) Shaping ability of rotatory or reciprocating instruments in curved canals: a micro-computed tomographic study. Brazilian Oral Research 30(1), 1-5.

Kashefinejad, M., Harandi, A., Eram, S., \& Bijani, A. (2016) Comparison of Single Visit Post Endodontic Pain Using Mtwo Rotary and Hand K-File Instruments: A Randomized Clinical Trial. Journal of Dentistry 13(1), 10-17.

Kfir, A., Rosenberg, E., \& Fuss, Z. (2006) Comparison in vivo of the first tapered and nontapered instruments that bind at the apical constriction. Oral Surgery, Oral Medicine, Oral Pathology, Oral Radiology, and Endodontology 102(3), 395-398.

Kfir, A., Rosenberg, E., Zuckerman, O., Tamse, A., \& Fuss, Z. (2003) Comparison of procedural errors resulting during root canal preparations completed by junior dental students in patients using an '8-step method' versus 'serial step-back technique'. International Endodontic Journal 36(1), $49-53$.

Khan, K. S., Daya, S., \& Jadad, A. (1996) The importance of quality of primary studies in producing unbiased systematic reviews. Archives of Internal Medicine 156(6), 661-666.

Kherlakian, D., Cunha, R., Ehrhardt, I., Zuolo, M., Kishen, A., \& Silveira, B. C. (2016) Comparison of the Incidence of Postoperative Pain after Using 2 Reciprocating Systems and a Continuous Rotary System: a Prospective Randomized Clinical Trial. Journal of Endodontics 42(2), $171-176$.

Krithikadatta, J., Sekar, V., Sudharsan, P., \& Velumurugan, N. (2016) Influence of three Ni-Ti cleaning and shaping files on postinstrumentation endodontic pain: A triple-blinded, randomized, controlled trial. Journal of Conservative Dentistry 19(4), 311-316.

Loguercio, A. D., Maran, B. M., Hanzen, T. A., Paula, A. M., Perdigao, J., \& Reis, A. (2017) Randomized clinical trials of dental bleaching - Compliance with the CONSORT Statement: a systematic review. Brazilian Oral Research 31(suppl 1), e60.

Marshall, F. J., \& Pappin, J. (1988) A crown-down pressureless preparation root canal enlargement technique. Portland, USA: Technique manual, University of Oregon Health Sciences University.

Mohammadi, Z., Jafarzadeh, H., Shalavi, S., \& Palazzi, F. (2017) Recent Advances in Root Canal Disinfection: A Review. Iranian Endodontic Journal 12(4), $402-406$.

Mollashahi, N. F., Saberi, E. A., Havaei, S. R., \& Sabeti, M. (2017) Comparison of Postoperative Pain after Root Canal Preparation with Two Reciprocating and Rotary Single-File Systems: A Randomized Clinical Trial. Iranian Endodontic Journal 12(1), 15-19.

Neelakantan, P., \& Sharma, S. (2015) Pain after single-visit root canal treatment with two single-file systems based on different kinematics--a prospective randomized multicenter clinical study. Clinical Oral Investigations 19(9), 2211-2217.

Nekoofar, M., Sheykhrezae, M., Meraji, N., Jamee, A., Shirvani, A., Jamee, J., \& Dummer, P. M. (2015) Comparison of the effect of root canal preparation by using WaveOne and ProTaper on postoperative pain: a randomized clinical trial. Journal of Endodontics 41(5), 575-578.

Neves, M. A. S., Provenzano, J. C., Rôças, I. N., \& Siqueira, J. F. (2016) Clinical Antibacterial Effectiveness of Root Canal Preparation with Reciprocating Single-instrument or Continuously Rotating Multi-instrument Systems. Journal of Endodontics 42(1), 25-29.

Pasqualini, D., Alovisi, M., Cemenasco, A., Mancini, L., Paolino, D. S., Bianchi, C. C., Roggia, A., Scotti, N., \& Berutti, E. (2015) Micro-Computed Tomography Evaluation of ProTaper Next and BioRace Shaping Outcomes in Maxillary First Molar Curved Canals. Journal of Endodontics 41(10), 17061710 .

Pasqualini, D., Corbella, S., Alovisi, M., Taschieri, S., Del Fabbro, M., Migliaretti, G., Carpegna, G. C., Scotti, N., \& Berutti, E. (2016) Postoperative quality of life following single-visit root canal treatment performed by rotary or reciprocating instrumentation: a randomized clinical trial. International Endodontic Journal 49(11), 1030-1039.

Pereira, A. S., Shitsuka, D. M., Parreira, F. J. \& Shitsuka, R. (2018) Metodologia da Pesquisa Científica. 1. ed. - Santa Maria, RS: UFSM, NTE, 119 p.

Peters, O. A., Barbakow, F., \& Peters, C. I. (2004) An analysis of endodontic treatment with three nickel-titanium rotary root canal preparation techniques. International Endodontic Journal 37(12), 849-859.

Pettiette, M. T., Delano, E. O., \& Trope, M. (2001) Evaluation of success rate of endodontic treatment performed by students with stainless-steel K-files and nickel-titanium hand files. Journal of Endodontics 27(2), 124-127. 
Pocock, S. (1983) Clinical Trials: A Practical Approach, Chichester UK: John Wiley \& Sons.

Reis, A., de Geus, J. L., Wambier, L., Schroeder, M., \& Loguercio, A. D. (2018) Compliance of Randomized Clinical Trials in Noncarious Cervical Lesions With the CONSORT Statement: A Systematic Review of Methodology. Operative Dentistry 43(3), E129-E151.

Relvas, J., Bastos, M., Marques, A., Garrido, A., \& Sponchiado, E. (2016) Assessment of postoperative pain after reciprocating or rotary NiTi instrumentation of root canals: a randomized, controlled clinical trial. Clinical Oral Investigations 20(8), 1987-1993.

Ritt, A. S., Buco, J., Wagner, M. H., Rosa, R. A., Vier-Pelisser, F. V., \& Só, M. V. R. (2012) Avaliação da eficácia da instrumentação manual x automatizada durante o retratamento endodôntico em canais radiculares obturados com guta-percha e cimento à base de hidróxido de cálcio. Revista da Faculdade de Odontologia UPF 17(1), 55-59.

Saha, S. G., Gupta, R. K., Bhardwaj, A., Misuriya, A., Saha, M. K., \& Nirwan, A. S. (2018) Comparison of the incidence of postoperative pain after using a continuous rotary system, a reciprocating system, and a Self-Adjusting File system in single-visit endodontics: A prospective randomized clinical trial. Journal of Conservative Dentistry 21(3), 333-338.

Sanfelice, C. M., da Costa, F. B., Reis Só, M. V., Vier-Pelisser, F., Souza Bier, C. A., \& Grecca, F. S. (2010) Effects of Four Instruments on Coronal Preenlargement by Using Cone Beam Computed Tomography. Journal of Endodontics 36(5), 858-861.

Saumya-Rajesh, P., Krithikadatta, J., Velmurugan, N., \& Sooriaprakas, C. (2017) Post-instrumentation pain after the use of either Mtwo or the SAF system: a randomized controlled clinical trial. International Endodontic Journal 50(8), 750-760.

Schriger, D. L., \& Altman, D. G. (2006) The content of medical journals instruction for authors. Annals of Internal Medicine 48(6), 743-749.

Schulz, K. F., Altman, D. G., \& Moher, D. (2010) CONSORT 2010 statement: Updated guidelines for reporting parallel group randomised trials. Journal of Pharmacology \& Pharmacotherapeutics 1, 100-107.

Schulz, K. F., Chalmers, I., Hayes, R. J., \& Altman, D. G. (1995) Empirical evidence of bias. Dimensions of methodological quality associated with estimates of treatment effects in controlled trials. JAMA 273(5), 408-412.

Shahi, S., Asghari, V., Rahimi, S., Lotfi, M., Samiei, M., Yavari, H., Shakouie, S., \& Nezafati, S. (2016) Postoperative Pain after Endodontic Treatment of Asymptomatic Teeth Using Rotary Instruments: A Randomized Clinical Trial. Iranian Endodontic Journal 11(1), 38-43.

Shokraneh, A., Ajami, M., Farhadi, N., Hosseini, M., \& Rohani, B. (2017) Postoperative endodontic pain of three different instrumentation techniques in asymptomatic necrotic mandibular molars with periapical lesion: a prospective, randomized, double-blind clinical trial. Clinical Oral Investigations 21(1), 413-418.

Topcuoglu, H., \& Topcuoglu, G. (2017) Postoperative pain after the removal of root canal filling material using different techniques in teeth with failed root canal therapy: a randomized clinical trial. Acta odontologica Scandinavica 75(4), 249-254.

Tortini, D., Colombo, M., \& Gagliani, M. (2007) Apical crown technique to model canal roots. A review of the literature. Minerva Stomatology 56(9), 445459.

Wan, N., Wang, X. Y., Qi, L., Yan, L., Ma, J., Yin, Y., Gao, W., \& Wu, P. (2017) Clinical evaluation of nickel-titanium mechanical instruments in three different movement patterns combined with warm vertical compaction in the single-visit endodontic treatment. Chinese Journal of Tissue Engineering Research 21, 893-898.

Wang, C., Xu, P., Ren, L., Dong, G., \& Ye, L. (2010) Comparison of post-obturation pain experience following one-visit and two-visit root canal treatment on teeth with vital pulps: a randomized controlled trial. International Endodontic Journal 43(8), 692-697.

Wang, Y., Zhu, Y., Wang, H., Han, J., He, Y., \& Zhu, M. (2010) Clinical effect of three kinds of rotary nickel-titanium instruments on root canal preparation of molars. Shanghai kou qiang yi xue 19(2), 118-123.

Wei, X., Lin, Z., \& Peng, S. (2003) The effect of root canal preparation with nickel-titanium rotary instruments in reducing post-operative pain. Hua xi kou qiang yi xue za zhi 21(3), 202-204.

Zhou, Z. J., \& He, H. (2013) Clinical study on the effects of single visit root canal treatment of chronic periapical periodontitis by two kinds of root canal preparation instruments system. Shanghai Kou Qiang Yi Xue 22(1), 85-88. 